\title{
Unique Existence Result of Approximate Solution to Initial Value Problem for Fractional Differential Equation of Variable Order Involving the Derivative Arguments on the Half-Axis
}

\author{
Shuqin Zhang ${ }^{1, *}$ and Lei $\mathrm{Hu}^{2}$ D \\ 1 Department of Mathematics, China University of Mining and Technology Beijing, \\ Ding No. 11 Xueyuan Road, Haidian District, Beijing 100083, China \\ 2 School of Science, Shandong Jiaotong University, Jinan 250023, China; huleimath@163.com \\ * Correspondence: zsqjk@163.com
}

Received: 29 December 2018; Accepted: 15 March 2019; Published: 20 March 2019

check for updates

\begin{abstract}
The semigroup properties of the Riemann-Liouville fractional integral have played a key role in dealing with the existence of solutions to differential equations of fractional order. Based on some results of some experts', we know that the Riemann-Liouville variable order fractional integral does not have semigroup property, thus the transform between the variable order fractional integral and derivative is not clear. These judgments bring us extreme difficulties in considering the existence of solutions of variable order fractional differential equations. In this work, we will introduce the concept of approximate solution to an initial value problem for differential equations of variable order involving the derivative argument on half-axis. Then, by our discussion and analysis, we investigate the unique existence of approximate solution to this initial value problem for differential equation of variable order involving the derivative argument on half-axis. Finally, we give examples to illustrate our results.
\end{abstract}

Keywords: variable order fractional derivative; initial value problem; fractional differential equations; piecewise constant functions; approximate solution

\section{Introduction}

In this paper, we will observe and study the unique existence of approximate solution to the following initial value problem of variable order

$$
\left\{\begin{array}{l}
D_{0+}^{p(t)} x(t)=f\left(t, x, D_{0+}^{q(t)} x\right), 0<t<+\infty \\
x(0)=0
\end{array}\right.
$$

where $0<q(t)<p(t)<1, f\left(t, x, D_{0+}^{q(t)} x\right)$ are given real functions, and $D_{0+}^{p(t)}, D_{0+}^{q(t)}$ denote derivatives of variable order $p(t)$ and $q(t)$ defined by

$$
\begin{aligned}
& D_{0+}^{p(t)} x(t)=\frac{d}{d t} \int_{0}^{t} \frac{(t-s)^{-p(t)}}{\Gamma(1-p(t))} x(s) d s, t>0 . \\
& D_{0+}^{q(t)} x(t)=\frac{d}{d t} \int_{0}^{t} \frac{(t-s)^{-q(t)}}{\Gamma(1-q(t))} x(s) d s, t>0,
\end{aligned}
$$

and $\frac{1}{\Gamma(1-p(t))} \int_{0}^{t}(t-s)^{-p(t)} x(s) d s$ is integral of variable order $1-p(t)$ for function $x(t)$, for details, please refer to [1]. 
The operators of variable order, which fall into a more complex category, are the derivatives and integrals whose orders are the functions of certain variables. There are several definitions of variable order fractional integrals and derivatives. The following are several definitions of variable order fractional integrals and derivatives, which can be found in [2]. Let $-\infty<a<b<\infty$.

Definition 1. Let $p:[a, b] \rightarrow(0,+\infty)$, the left Riemann-Liouville fractional integral of order $\alpha(t)$ for function $x(t)$ are defined as the following two types

$$
\begin{aligned}
& I_{a+}^{\alpha(t)} x(t)=\int_{a}^{t} \frac{(t-s)^{\alpha(t)-1}}{\Gamma(\alpha(t))} x(s) d s, t>a, \\
& I_{a+}^{\alpha(t)} x(t)=\int_{a}^{t} \frac{(t-s)^{\alpha(s)-1}}{\Gamma(\alpha(s))} x(s) d s, t>a .
\end{aligned}
$$

Definition 2. Let $\alpha:[a, b] \rightarrow(n-1, n]$ ( $n$ is a natural number), the left Riemann-Liouville fractional derivative of order $\alpha(t)$ for function $x(t)$ are defined as the following two types

$$
\begin{aligned}
& D_{a+}^{\alpha(t)} x(t)=\left(\frac{d}{d t}\right)^{n} \int_{a}^{t} \frac{(t-s)^{n-\alpha(t)-1}}{\Gamma(n-\alpha(t))} x(s) d s, t>a, \\
& D_{a+}^{\alpha(t)} x(t)=\left(\frac{d}{d t}\right)^{n} \int_{a}^{t} \frac{(t-s)^{n-\alpha(s)-1}}{\Gamma(n-\alpha(s))} x(s) d s, t>a .
\end{aligned}
$$

Definition 3. Let $\alpha:[a, b] \rightarrow(n-1, n]$ ( $n$ is a natural number), the left Caputo fractional derivative of order $\alpha(t)$ for function $x(t)$ are defined as the following two types

$$
\begin{aligned}
& { }^{C} D_{a+}^{\alpha(t)} x(t)=\int_{a}^{t} \frac{(t-s)^{n-\alpha(t)-1}}{\Gamma(n-\alpha(t))} x^{(n)}(s) d s, t>a, \\
& { }^{C} D_{a+}^{\alpha(t)} x(t)=\int_{a}^{t} \frac{(t-s)^{n-\alpha(s)-1}}{\Gamma(n-\alpha(s))} x^{(n)}(s) d s, t>a .
\end{aligned}
$$

The problems denoted by the operator of variable order are apparently more complicated than the ones denoted by the operator of constant order. Recently, some authors have considered the applications of derivatives of variable order in various sciences such as anomalous diffusion modeling, mechanical applications, multi-fractional Gaussian noises. Among these, there have been many works dealing with numerical methods for some class of variable order fractional differential equations, for instance, [1-20].

We notice that, if the order $p(t)$ is a constant function $q$, then the Riemann-Liouville variable order fractional derivatives and integrals are the Riemann-Liouville fractional derivative and integral, respectively [21]. We know there are some important properties as following. Let $-\infty<b<\infty$.

Lemma 1. [21] The Riemann-Liouville fractional integral defined for function $x(t) \in L(0, b)$ exists almost everywhere.

Lemma 2. [21] The equality $I_{0+}^{\gamma} I_{0+}^{\delta} x(t)=I_{0+}^{\delta} I_{0+}^{\gamma} x(t)=I_{0+}^{\gamma+\delta} x(t), 0<\gamma<1,0<\delta<1$ holds for $x \in L(0, b)$.

Lemma 2 is semigroup property for the Riemann-Liouville fractional integral, which is very crucial in obtaining the following Lemmas 3-5. In other words, without Lemma 2, one could not have Lemmas 3-5, for details, please refer to [21].

Lemma 3. [21] The equality $D_{0+}^{\gamma} I_{0+}^{\gamma} x(t)=x(t), 0<\gamma<1$ holds for $x \in L(0, b)$. 
Lemma 4. [21] Let $0<\alpha<1$, then the differential equation

$$
D_{0+}^{\alpha} x=0, t>0
$$

has solution

$$
x(t)=c t^{\alpha-1}, c \in R
$$

Lemma 5. [21] Let $0<\alpha<1, x \in L(0, b), D_{0+}^{\alpha} x \in L(0, b)$. Then the following equality holds

$$
I_{0+}^{\alpha} D_{0+}^{\alpha} x(t)=x(t)+c t^{\alpha-1}, c \in R
$$

These properties play a very important role in considering the existence of the solutions of differential equations for the Riemann-Liouville fractional derivative, for details, please refer to [22-26]. However, from [15-18], for general functions $h(t), g(t)$, we notice that the semigroup property does not hold, i.e., $I_{a+}^{h(t)} I_{a+}^{g(t)} \neq I_{a+}^{h(t)+g(t)}$. Thus, it brings us extreme difficulties, that we cannot get these properties like Lemmas 3-5 for the variable order fractional operators (integral and derivative). Without these properties for variable order fractional derivative and integral, we can hardly consider the existence of solutions of differential equations for variable order derivative by means of nonlinear functional analysis (for instance, some fixed point theorems).

In [18], by means of Banach contraction principle, we considered the uniqueness result of solutions to initial value problems of differential equations of variable order

$$
\left\{\begin{array}{l}
D_{0+}^{q(t)} x(t)=f(t, x), 0<t \leq T \\
x(0)=0
\end{array}\right.
$$

where $0<T<+\infty, D_{0+}^{q(t)}$ denotes derivative of variable order defined by (2), and $q:[0, T] \rightarrow(0,1]$ is a piecewise constant function with partition $P=\left\{\left[0, T_{1}\right],\left(T_{1}, T_{2}\right],\left(T_{2}, T_{3}\right], \cdots,\left(T_{N^{*}-1}, T\right]\right\}\left(N^{*}\right.$ is a given natural number) of the finite interval $[0, T]$, i.e.,

$$
q(t)=\sum_{k=1}^{N^{*}} q_{k} I_{k}(t), t \in[0, T],
$$

where $0<q_{k} \leq 1, k=1,2, \cdots, N^{*}$ are constants, and $I_{k}$ is the indicator of the interval $\left[T_{k-1}, T_{k}\right]$, $k=1,2, \cdots, N^{*}$ (here $T_{0}=0, T_{N^{*}}=T$ ), that is $I_{k}=1$ for $t \in\left[T_{k-1}, T_{k}\right], I_{k}=0$ for elsewhere.

In this paper, we will consider the existence of solutions to the problem (1) for variable orders $p(t), q(t)$ are not piecewise constants. Based on some analysis, we will introduce the concept of approximate solution to the problem (1). Then, according to our discussion and analysis, we explore the unique existence of the approximate solution of the problem (1).

This paper is organized as follows. In Section 2, we provide some facts to the variable order integral and derivative through several examples. Also, we state some results which will play a very important role in obtaining our main results. In Section 3, we set forth our main result. Finally, two examples are given.

\section{Some Preliminaries on Approximate Solution}

In this section, we give some preliminaries on approximate solutions to the initial value problem (1). First of all, we use an example to illustrate the claim: for general function $p(t), q(t)$, the Riemann-Liouville variable order fractional integral does not have the semigroup property.

Example 1. Let $p(t)=\frac{t}{6}+\frac{1}{3}, q(t)=\frac{t}{4}+\frac{1}{4}, f(t)=1,0 \leq t \leq 3$. Now, we calculate $\left.I_{0+}^{p(t)} I_{0+}^{q(t)} f(t)\right|_{t=1}$ and $\left.I_{0+}^{p(t)+q(t)} f(t)\right|_{t=1}$ which are defined in (3). 
For $1 \leq t \leq 3$, we have

$$
\begin{aligned}
I_{0+}^{p(t)} I_{0+}^{q(t)} f(t) & =\int_{0}^{t} \frac{(t-s)^{\frac{t}{6}+\frac{1}{3}-1}}{\Gamma\left(\frac{t}{6}+\frac{1}{3}\right)} \int_{0}^{s} \frac{(s-\tau)^{\frac{s}{4}+\frac{1}{4}-1}}{\Gamma\left(\frac{s}{4}+\frac{1}{4}\right)} d \tau d s \\
& =\int_{0}^{t} \frac{(t-s)^{\frac{t}{6}-\frac{2}{3}} s^{\frac{1}{4}+\frac{s}{4}}}{\Gamma\left(\frac{t}{6}+\frac{1}{3}\right) \Gamma\left(\frac{5}{4}+\frac{s}{4}\right)} d s \\
& =\int_{0}^{1} \frac{(t-s)^{\frac{t}{6}-\frac{2}{3}} s^{\frac{1}{4}+\frac{s}{4}}}{\Gamma\left(\frac{t}{6}+\frac{1}{3}\right) \Gamma\left(\frac{5}{4}+\frac{s}{4}\right)} d s+\int_{1}^{t} \frac{(t-s)^{\frac{t}{6}-\frac{2}{3}} s^{\frac{1}{4}+\frac{s}{4}}}{\Gamma\left(\frac{t}{6}+\frac{1}{3}\right) \Gamma\left(\frac{5}{4}+\frac{s}{4}\right)} d s .
\end{aligned}
$$

We set $M_{1}=\max _{1 \leq t \leq 3}\left|\frac{1}{\Gamma(p(t))}\right|$ and $M_{2}=\max _{1 \leq s \leq 3}\left|\frac{1}{\Gamma\left(\frac{5}{4}+\frac{s}{4}\right)}\right|$. For $1 \leq t \leq 3$, it holds

$$
\begin{aligned}
\left|\int_{1}^{t} \frac{(t-s)^{\frac{t}{6}-\frac{2}{3}} s^{\frac{1}{4}+\frac{s}{4}}}{\Gamma\left(\frac{t}{6}+\frac{1}{3}\right) \Gamma\left(\frac{5}{4}+\frac{s}{4}\right)} d s\right| & =\left|\int_{1}^{t} 3^{\frac{t}{6}-\frac{2}{3}}\left(\frac{t-s}{3}\right)^{\frac{t}{6}-\frac{2}{3}} \frac{s^{\frac{1}{4}+\frac{s}{4}}}{\Gamma\left(\frac{t}{6}+\frac{1}{3}\right) \Gamma\left(\frac{5}{4}+\frac{s}{4}\right)} d s\right| \\
& \leq \quad M_{1} M_{2} \int_{1}^{t} 3^{\frac{1}{2}-\frac{2}{3}}\left(\frac{t-s}{3}\right)^{\frac{1}{6}-\frac{2}{3}} s d s \\
& \leq M_{1} M_{2} \int_{1}^{t} 3^{\frac{1}{3}}(t-s)^{-\frac{1}{2}} 3 d s \\
& =2 \times 3^{\frac{4}{3}} M_{1} M_{2}(t-1)^{\frac{1}{2}},
\end{aligned}
$$

hence, we have

$$
\left[\int_{1}^{t} \frac{(t-s)^{\frac{t}{6}-\frac{2}{3}} s^{\frac{1}{4}+\frac{s}{4}}}{\Gamma\left(\frac{t}{6}+\frac{1}{3}\right) \Gamma\left(\frac{5}{4}+\frac{s}{4}\right)} d s\right]_{t=1}=0
$$

So, we get

$$
\left.I_{0+}^{p(t)} I_{0+}^{q(t)} f(t)\right|_{t=1}=\int_{0}^{1} \frac{(1-s)^{-\frac{1}{2}} s^{\frac{1}{4}+\frac{s}{4}}}{\Gamma\left(\frac{1}{2}\right) \Gamma\left(\frac{5}{4}+\frac{s}{4}\right)} d s \approx 1.063
$$

and

$$
\left.I_{0+}^{p(t)+q(t)} f(t)\right|_{t=1}=\int_{0}^{1} \frac{(1-s)^{p(1)+q(1)-1}}{\Gamma(p(1)+q(1))} d s=\int_{0}^{1} d s=1
$$

Therefore,

$$
\left.I_{0+}^{p(t)} I_{0+}^{q(t)} f(t)\right|_{t=1} \neq\left. I_{0+}^{p(t)+q(t)} f(t)\right|_{t=1}
$$

Without the semigroup property of the Riemann-Liouville variable order fractional integral, we can assure that the variable order fractional integration operator of non-constant continuous functions $p(t)$ for $x(t)$ does not have the properties like Lemmas 3-5. Consequently, we cannot transform differential equations of variable order into an integral equation.

Let $L[x(t) ; s], L\left[I_{0+}^{p(t)} x(t) ; s\right], L\left[D_{0+}^{p(t)} x(t) ; s\right]$ denote the Laplace transforms of functions $x(t)$, $I_{0+}^{p(t)} x(t)$ and $D_{0+}^{p(t)} x(t)$. We have not found out the explicit connection between $L[x(t) ; s]$ and $L\left[I_{0+}^{p(t)} x(t) ; s\right]$, as a result, we have not found out the explicit connection between $L[x(t) ; s]$ and $L\left[D_{0+}^{p(t)} x(t) ; s\right]$.

Example 2. Let $p(t)=\frac{1}{\sqrt{ } t+1}, t \geq 0$. We consider the Laplace transforms of functions $t(t \geq 0)$ and $I_{0+}^{p(t)} t(t \geq$ 0) defined in (3). We can know that

$$
L[t ; s]=\int_{0}^{\infty} e^{-s t} t d t=\frac{1}{s^{2}}
$$




$$
\begin{aligned}
L\left[I_{0+}^{(t+1)^{-\frac{1}{2}}} t ; s\right] & =\int_{0}^{\infty} e^{-s t} \int_{0}^{t} \frac{(t-\tau)^{(t+1)^{-\frac{1}{2}}-1}}{\Gamma\left((t+1)^{-\frac{1}{2}}\right)} \tau d \tau d t \\
& =\int_{0}^{\infty} e^{-s t} \int_{\tau}^{\infty} \frac{(t-\tau)^{(t+1)^{-\frac{1}{2}}-1}}{\Gamma\left((t+1)^{-\frac{1}{2}}\right)} \tau d t d \tau \\
& =\int_{0}^{\infty} e^{-s(\tau+r)} \int_{0}^{\infty} \frac{r^{(\tau+r+1)^{-\frac{1}{2}}-1}}{\Gamma\left((\tau+r+1)^{-\frac{1}{2}}\right)} \tau d r d \tau \\
& =\int_{0}^{\infty} e^{-s \tau} \tau \int_{0}^{\infty} e^{-s r} \frac{r^{(\tau+r+1)^{-\frac{1}{2}}-1}}{\Gamma\left((\tau+r+1)^{-\frac{1}{2}}\right)} d r d \tau
\end{aligned}
$$

By (10) or (11), we do not get the explicit connection between $L[t ; s]$ and $L\left[I_{0+}^{(t+1)^{-\frac{1}{2}}} t ; s\right]$.

In view of this example, the definition of variable order fractional derivative and the connection between the Laplace transforms of function $x(t)$ and its derivative $x^{\prime}(t)$, we cannot obtain the Laplace transform formula for variable order fractional derivatives (2). Based on these facts, we cannot get the explicit expression of the solutions for the problem (1).

Throughout this paper, we assume that

$\left(A_{1}\right)$ Let $p:[0,+\infty) \rightarrow(0,1)$ and $q:[0,+\infty) \rightarrow(0,1)$ be continuous functions, $q(t)<p(t)$ for all $t \in[0,+\infty)$, and that $p(t), q(t)$ satisfy

$$
\lim _{t \rightarrow+\infty} p(t)=\rho_{1}, \lim _{t \rightarrow+\infty} q(t)=\rho_{2}, 0<\rho_{1}, \rho_{2}<1 .
$$

The following result is necessary in our next analysis of main result.

Lemma 6. Let condition $\left(A_{1}\right)$ hold. Then there exist positive constant $T$, natural number $n^{*}$ and intervals $\left[0, T_{1}\right],\left(T_{1}, T_{2}\right], \cdots,\left(T_{n^{*}-1}, T\right](T,+\infty)\left(n^{*} \in N\right)$ and functions $\alpha:[0,+\infty) \rightarrow(0,1)$ and $\beta:[0,+\infty) \rightarrow$ $(0,1)$ defined by

$$
\begin{aligned}
& \alpha(t)=\sum_{k=1}^{n^{*}} p_{k} I_{k}(t)+\rho_{1} I_{T}(t), \quad t \in[0,+\infty), \\
& \beta(t)=\sum_{k=1}^{n^{*}} q_{k} I_{k}(t)+\rho_{2} I_{T}(t), \quad t \in[0,+\infty),
\end{aligned}
$$

where $p_{k}, q_{k} \in(0,1), I_{k}(t)$ is the indicator of the interval $\left[T_{k-1}, T_{k}\right]\left(k=1,2, \cdots, n^{*}\right.$, here $\left.T_{0}=0, T_{n^{*}}=T\right)$, i.e., $I_{k}(t)=1$ for $t \in\left[T_{k-1}, T_{k}\right], I_{k}(t)=0$ for t lying in elsewhere; $I_{T}(t)$ is the indicator of interval $(T,+\infty)$, i.e., $I_{T}(t)=1$ for $t \in(T,+\infty), I_{T}(t)=0$ for $t$ lying in elsewhere, such that for arbitrary small $\varepsilon>0$,

$$
|p(t)-\alpha(t)|<\varepsilon,|q(t)-\beta(t)|<\varepsilon, \quad 0 \leq t<+\infty .
$$

Proof. By (12), for $\forall \varepsilon>0$, there exist $\bar{T}_{1}, \bar{T}_{2}>0$, such that

$$
\left|p(t)-\rho_{1}\right|<\varepsilon, t>\bar{T}_{1} ;\left|p(t)-\rho_{2}\right|<\varepsilon, t>\bar{T}_{2} .
$$

Let $T=\max \left\{\bar{T}_{1}, \bar{T}_{2}\right\}$, then, for $\forall \varepsilon>0$, we have that

$$
\left|p(t)-\rho_{1}\right|<\varepsilon,\left|p(t)-\rho_{2}\right|<\varepsilon, t>T .
$$

We know that $p:[0, T] \rightarrow(0,1), q:[0, T] \rightarrow(0,1)$ are continuous functions. Since $p(t)$ is right continuous at point 0 , then, for arbitrary small $\varepsilon>0$, there is $\delta_{01}>0$ such that

$$
|p(t)-p(0)|<\varepsilon \text {, for } 0 \leq t \leq \delta_{01} .
$$


Since $q(t)$ is right continuous at point 0 , then, for arbitrary small $\varepsilon>0$, there is $\delta_{02}>0$ such that

$$
|q(t)-q(0)|<\varepsilon, \text { for } 0 \leq t \leq \delta_{02} .
$$

Then for arbitrary small $\varepsilon>0$, takeing $\delta_{0}=\min \left\{\delta_{01}, \delta_{02}\right\}$, it holds

$$
|p(t)-p(0)|<\varepsilon,|q(t)-q(0)|<\varepsilon, \text { for } 0 \leq t \leq \delta_{0} .
$$

We take point $\delta_{0} \doteq T_{1}$ (if $T_{1}<T$, we consider continuities of $p(t), q(t)$ at point $T_{1}$, otherwise, we end this procedure). Since $p(t)$ is right continuous at point $T_{1}$, so, for arbitrary small $\varepsilon>0$, there is $\delta_{11}>0$ such that

$$
\left|p(t)-p\left(T_{1}\right)\right|<\varepsilon, \text { for } T_{1} \leq t \leq T_{1}+\delta_{11},
$$

Since $q(t)$ is right continuous at point $T_{1}$, then, for arbitrary small $\varepsilon>0$, there is $\delta_{12}>0$ such that

$$
\left|q(t)-q\left(T_{1}\right)\right|<\varepsilon, \text { for } T_{1} \leq t \leq T_{1}+\delta_{12} .
$$

Hence, for arbitrary small $\varepsilon>0$, taking $\delta_{1}=\min \left\{\delta_{11}, \delta_{12}\right\}$, it holds

$$
\left|p(t)-p\left(T_{1}\right)\right|<\varepsilon,\left|q(t)-q\left(T_{1}\right)\right|<\varepsilon, \text { for } T_{1} \leq t \leq T_{1}+\delta_{1} .
$$

We take point $T_{1}+\delta_{1} \doteq T_{2}$ (if $T_{2}<T$, we consider continuities of $p(t), q(t)$ at point $T_{2}$, otherwise, we end this procedure). Since $p(t)$ is right continuous at point $T_{2}$, so, for arbitrary small $\varepsilon>0$, there is $\delta_{21}>0$ such that

$$
\left|p(t)-p\left(T_{2}\right)\right|<\varepsilon, \text { for } T_{2} \leq t \leq T_{2}+\delta_{21} .
$$

Since $q(t)$ is right continuous at point $T_{2}$, so, for arbitrary small $\varepsilon>0$, there is $\delta_{22}>0$ such that

$$
\left|q(t)-q\left(T_{2}\right)\right|<\varepsilon, \text { for } T_{2} \leq t \leq T_{2}+\delta_{22} .
$$

Thus, for arbitrary small $\varepsilon>0$, taking $\delta_{2}=\min \left\{\delta_{21}, \delta_{22}\right\}$, it holds

$$
\left|p(t)-p\left(T_{2}\right)\right|<\varepsilon,\left|q(t)-q\left(T_{2}\right)\right|<\varepsilon, \text { for } T_{2} \leq t \leq T_{2}+\delta_{2} .
$$

We take point $T_{2}+\delta_{2} \doteq T_{3}$ (if $T_{3}<T$, we consider continuities of $p(t), q(t)$ at point $T_{3}$, otherwise, we end this procedure). Since $p(t)$ is right continuous at point $T_{3}$, so, for arbitrary small $\varepsilon>0$, there is $\delta_{31}>0$ such that

$$
\left|p(t)-p\left(T_{3}\right)\right|<\varepsilon, \text { for } T_{3} \leq t \leq T_{3}+\delta_{31},
$$

Since $q(t)$ is right continuous at point $T_{3}$, so, for arbitrary small $\varepsilon>0$, there is $\delta_{32}>0$ such that

$$
\left|q(t)-q\left(T_{3}\right)\right|<\varepsilon, \text { for } T_{3} \leq t \leq T_{3}+\delta_{32} .
$$

Therefore, for arbitrary small $\varepsilon>0$, taking $\delta_{3}=\min \left\{\delta_{31}, \delta_{32}\right\}$, it holds

$$
\left|p(t)-p\left(T_{3}\right)\right|<\varepsilon, \quad\left|q(t)-q\left(T_{3}\right)\right|<\varepsilon, \text { for } T_{3} \leq t \leq T_{3}+\delta_{3} .
$$

Since $[0, T]$ is a finite interval, then, continuing this analysis procedure, we could obtain that there exist $\delta_{n^{*}-2}>0, \delta_{n^{*}-1}>0\left(n^{*} \in N\right)$ such that $T_{n^{*}-2}+\delta_{n^{*}-2} \doteq T_{n^{*}-1}<T, T_{n^{*}-1}+\delta_{n^{*}-1} \geq T$, such that for arbitrary small $\varepsilon>0$, it holds

$$
\left|p(t)-p\left(T_{n^{*}-1}\right)\right|<\varepsilon,\left|q(t)-q\left(T_{n^{*}-1}\right)\right|<\varepsilon \text { for } T_{n^{*}-1} \leq t \leq T,
$$


From (16)-(21), we could let

$$
\begin{aligned}
& p(0) \doteq p_{1}, p\left(T_{1}\right) \doteq p_{2}, p\left(T_{2}\right) \doteq p_{3}, p\left(T_{3}\right) \doteq p_{4}, \cdots, p\left(T_{n^{*}-1}\right) \doteq p_{n^{*}} \\
& q(0) \doteq q_{1}, q\left(T_{1}\right) \doteq q_{2}, q\left(T_{2}\right) \doteq q_{3}, q\left(T_{3}\right) \doteq q_{4}, \cdots, q\left(T_{n^{*}-1}\right) \doteq q_{n^{*}}
\end{aligned}
$$

Thus, we define functions $\alpha, \beta:[0,+\infty) \rightarrow(0,1)$ as following

$$
\alpha(t)=\left\{\begin{array}{l}
p_{1}, \quad t \in\left[0, T_{1}\right], \\
p_{2}, \quad t \in\left(T_{1}, T_{2}\right], \\
\vdots \\
p_{n^{*}}, \quad t \in\left(T_{n^{*}-1}, T\right], \\
\rho_{1}, \quad t \in(T,+\infty),
\end{array} \quad \beta(t)=\left\{\begin{array}{l}
q_{1}, \quad t \in\left[0, T_{1}\right], \\
q_{2}, t \in\left(T_{1}, T_{2}\right], \\
\vdots \\
q_{n^{*}}, \quad t \in\left(T_{n^{*}-1}, T\right] \\
\rho_{2}, \quad t \in(T,+\infty) .
\end{array}\right.\right.
$$

Hence, from the previous arguments, for arbitrary small $\varepsilon>0$, we have

$$
\left\{\begin{array}{l}
\left|p(t)-p_{1}\right|<\varepsilon,\left|q(t)-q_{1}\right|<\varepsilon, \text { for } t \in\left[0, T_{1}\right] \\
\left|p(t)-p_{2}\right|<\varepsilon,\left|q(t)-q_{2}\right|<\varepsilon, \text { for } t \in\left(T_{1}, T_{2}\right] \\
\vdots \\
\left|p(t)-p_{n^{*}}\right|<\varepsilon,\left|q(t)-q_{n^{*}}\right|<\varepsilon, \text { for } t \in\left(T_{n^{*}-1}, T\right] \\
\left|p(t)-\rho_{1}\right|<\varepsilon,\left|q(t)-\rho_{2}\right|<\varepsilon, \text { for } t \in(T,+\infty) .
\end{array}\right.
$$

Thus, we complete this proof.

The following example illustrates that the semigroup property of the variable order fractional integral does not holds for the piecewise constant functions $p(t)$ and $q(t)$ defined in the same partition of finite interval $[a, b]$.

Example 3. Let $p(t)=\left\{\begin{array}{l}4,0 \leq t \leq 1, \\ 3,1<t \leq 4,\end{array} q(t)=\left\{\begin{array}{l}3,0 \leq t \leq 1, \\ 2,1<t \leq 4,\end{array}\right.\right.$ and $f(t)=1,0 \leq t \leq 4$. We'll verify $\left.I_{0+}^{p(t)} I_{0+}^{q(t)} f(t)\right|_{t=3} \neq\left. I_{0+}^{p(t)+q(t)} f(t)\right|_{t=3}$, here, the variable order fractional integral is defined in (3). For $1 \leq t \leq 4$, we have

$$
\begin{aligned}
& I_{0+}^{p(t)} I_{0+}^{q(t)} f(t) \\
= & \int_{0}^{1} \frac{(t-s)^{p(t)-1}}{\Gamma(p(t))} \int_{0}^{s} \frac{(s-\tau)^{3-1}}{\Gamma(3)} d \tau d s+\int_{1}^{t} \frac{(t-s)^{p(t)-1}}{\Gamma(p(t))} \int_{0}^{s} \frac{(s-\tau)^{2-1}}{\Gamma(2)} d \tau d s \\
= & \int_{0}^{1} \frac{(t-s)^{p(t)-1} s^{3}}{6 \Gamma(p(t))} d s+\int_{1}^{t} \frac{(t-s)^{p(t)-1} s^{2}}{2 \Gamma(2) \Gamma(p(t))} d s,
\end{aligned}
$$

thus, we have

$$
\left.I_{0+}^{p(t)} I_{0+}^{q(t)} f(t)\right|_{t=3}=\quad \int_{0}^{1} \frac{(3-s)^{2} s^{3}}{6 \Gamma(3)} d s+\int_{1}^{3} \frac{(3-s)^{2} s^{2}}{2 \Gamma(2) \Gamma(3)} d s=\frac{245}{144}
$$




$$
\left.I_{0+}^{p(t)+q(t)} f(t)\right|_{t=3}=\int_{0}^{3} \frac{(3-s)^{p(3)+q(3)-1}}{\Gamma(p(3)+q(3))} d s=\frac{3^{3+2}}{\Gamma(1+3+2)}=\frac{81}{40} .
$$

Therefore, we obtain

$$
\left.I_{0+}^{p(t)} I_{0+}^{q(t)} f(t)\right|_{t=3} \neq\left. I_{0+}^{p(t)+q(t)} f(t)\right|_{t=3},
$$

which implies that the semigroup property of the variable order fractional integral does not hold for the piecewise constant functions $p(t)$ and $q(t)$ defined in the same partition $[0,1],(1,4]$ of finite interval $[0,4]$.

Lemma 7. [10] Suppose $\beta>0, a(t)$ is a nonnegative nondecreasing function locally integrable on $0 \leq t<L$ (some $L \leq+\infty$ ) and $g(t)$ is a nonnegative nondecreasing continuous function defined on $0 \leq t<L, g(t) \leq M$ (constant), and suppose $u(t)$ is nonnegative and locally integrable on $0 \leq t<L$ with

$$
u(t) \leq a(t)+g(t) \int_{0}^{t}(t-s)^{\beta-1} u(s) d s
$$

on this interval. Then

$$
u(t) \leq a(t) E_{\beta}\left(g(t) \Gamma(\beta) t^{\beta}\right), 0 \leq t<L,
$$

where $E_{\beta}$ is the Mittag-Leffler function defined by $E_{\beta}(z)=\sum_{k=0}^{\infty} \frac{z^{k}}{\Gamma(k \beta+1)}$.

\section{Existence of Approximate Solution}

According to the previous arguments, we do not transform the problem (1) into an integral equation. Here, we consider the unique existence of approximate solution of the problem (1). In this section, we present our main results.

Now we make the following assumptions:

$\left(A_{2}\right) f:[0,+\infty) \times R^{2} \rightarrow R$ be a continuous function, and there exist positive constants $\lambda>\left\{\rho_{1}, \rho_{2}\right\}$, $c_{1}, c_{2}>0$ satisfying

$$
\frac{c_{1}}{\Gamma\left(1+\rho_{1}\right)}+\frac{c_{2}}{\Gamma\left(1+\rho_{1}-\rho_{2}\right)}<1
$$

such that

$$
\left|f\left(t,\left(1+t^{\lambda}\right) x_{1},\left(1+t^{\lambda}\right) y_{1}\right)-f\left(t,\left(1+t^{\lambda}\right) x_{2},\left(1+t^{\lambda}\right) y_{2}\right)\right| \leq c_{1}\left|x_{1}-x_{2}\right|+c_{2}\left|y_{1}-y_{2}\right|,
$$

where $\rho_{1}, \rho_{2}$ are the constants in $(A 1)$.

$\left(A_{3}\right) f(t, 0,0)(t \in(0,+\infty))$ satisfies

$$
\lim _{t \rightarrow+\infty} \frac{1}{1+t^{\lambda}} \int_{0}^{t}(t-s)^{\rho_{1}-\rho_{2}-1}|f(s, 0,0)| d s=0 .
$$

Let $B_{i}$ denote the Banach spaces defined as

$$
B_{i}=\left\{x \mid x \in C\left[0, T_{i}\right]\right\}
$$

with the norm

$$
\|x\|_{B_{i}}=\max _{t \in\left[0, T_{i}\right]}|x(t)|,
$$

where $T_{i}$ is the constant obtained in Lemma $6, i=1, \cdots, n^{*}\left(T_{n^{*}}=T\right)$. Let

$$
E=\left\{x \mid x \in C[0,+\infty), \sup _{t \geq 0} \frac{|x(t)|}{1+t^{\lambda}}<\infty\right\}
$$


with the norm

$$
\|x\|_{E}=\sup _{t \geq 0} \frac{|x(t)|}{1+t^{\lambda}}
$$

where $\lambda>\left\{\rho_{1}, \rho_{2}\right\}$. Then, by the same arguments as in Lemma 2.2 of [22], we know that $\left(E,\|\cdot\|_{E}\right)$ is a Banach space, here we omit this proof.

Now, we consider the following initial value problem

$$
\left\{\begin{array}{l}
D_{0+}^{\alpha(t)} x(t)=f\left(t, x, D_{0+}^{\beta(t)} x\right), 0<t<+\infty \\
x(0)=0
\end{array}\right.
$$

where $\alpha(t), \beta(t)$ are defined in (13) and (14).

In order to obtain our main results, we start off by carrying on essential analysis to the equation of (26).

By (13) and (14), we get

$$
\begin{gathered}
\int_{0}^{t} \frac{(t-s)^{-\alpha(t)}}{\Gamma(1-\alpha(t))} x(s) d s=\sum_{k=1}^{n^{*}} I_{k}(t) \int_{0}^{t} \frac{(t-s)^{-p_{k}}}{\Gamma\left(1-p_{k}\right)} x(s) d s+I_{T}(t) \int_{0}^{t} \frac{(t-s)^{-\rho_{1}}}{\Gamma\left(1-\rho_{1}\right)} x(s) d s, \\
\int_{0}^{t} \frac{(t-s)^{-\beta(t)}}{\Gamma(1-\beta(t))} x(s) d s=\sum_{k=1}^{n^{*}} I_{k}(t) \int_{0}^{t} \frac{(t-s)^{-q_{k}}}{\Gamma\left(1-q_{k}\right)} x(s) d s+I_{T}(t) \int_{0}^{t} \frac{(t-s)^{-\rho_{2}}}{\Gamma\left(1-\rho_{2}\right)} x(s) d s \doteq h_{\beta, x}(t),
\end{gathered}
$$

So, the equation of (26) can be written by

$$
\frac{d}{d t}\left(\sum_{k=1}^{n^{*}} I_{k}(t) \int_{0}^{t} \frac{(t-s)^{-p_{k}}}{\Gamma\left(1-p_{k}\right)} x(s) d s+I_{T}(t) \int_{0}^{t} \frac{(t-s)^{-\rho_{1}}}{\Gamma\left(1-\rho_{1}\right)} x(s) d s\right)=f\left(t, x, \frac{d}{d t} h_{\beta, x}(t)\right), 0<t<+\infty .
$$

Then, Equation (27) in the interval $\left(0, T_{1}\right]$ can be written by

$$
\frac{d}{d t} \int_{0}^{t} \frac{(t-s)^{-p_{1}}}{\Gamma\left(1-p_{1}\right)} x(s) d s=D_{0+}^{p_{1}} x(t)=f\left(t, x, D_{0+}^{q_{1}} x\right), 0<t \leq T_{1} .
$$

The Equation (27) in the interval $\left(T_{1}, T_{2}\right]$ can be written by

$$
\frac{d}{d t} \int_{0}^{t} \frac{(t-s)^{-p_{2}}}{\Gamma\left(1-p_{2}\right)} x(s) d s=f\left(t, x, \frac{d}{d t} \int_{0}^{t} \frac{(t-s)^{-q_{2}}}{\Gamma\left(1-q_{2}\right)} x(s) d s\right), \quad T_{1}<t \leq T_{2} .
$$

The Equation (27) in the interval $\left(T_{2}, T_{3}\right]$ can be written by

$$
\frac{d}{d t} \int_{0}^{t} \frac{(t-s)^{-p_{3}}}{\Gamma\left(1-p_{3}\right)} x(s) d s=f\left(t, x, \frac{d}{d t} \int_{0}^{t} \frac{(t-s)^{-q_{3}}}{\Gamma\left(1-q_{3}\right)} x(s) d s\right), \quad T_{2}<t \leq T_{3} .
$$

The Equation (27) in the interval $\left(T_{i-1}, T_{i}\right], i=4,5, \cdots, n^{*}\left(T_{n^{*}}=T\right)$ can be written by

$$
\frac{d}{d t} \int_{0}^{t} \frac{(t-s)^{-p_{i}}}{\Gamma\left(1-p_{i}\right)} x(s) d s=f\left(t, x, \frac{d}{d t} \int_{0}^{t} \frac{(t-s)^{-q_{i}}}{\Gamma\left(1-q_{i}\right)} x(s) d s\right), \quad T_{i-1}<t \leq T_{i} .
$$

The Equation (27) in the interval $(T,+\infty)$ can be written by

$$
\frac{d}{d t} \int_{0}^{t} \frac{(t-s)^{-\rho_{1}}}{\Gamma\left(1-\rho_{1}\right)} x(s) d s=f\left(t, x, \frac{d}{d t} \int_{0}^{t} \frac{(t-s)^{-\rho_{2}}}{\Gamma\left(1-\rho_{2}\right)} x(s) d s\right), \quad T<t<+\infty .
$$

Now, we present the definition of a solution to the problem (26), which is crucial in our work. 
Definition 4. We say the problem (26) exists one unique solution, if there are unique functions $u_{i}(t)$, $i=1,2, \cdots, n^{*}$, such that $u_{1} \in C\left[0, T_{1}\right]$ satisfying Equation (28) and $u_{1}(0)=0 ; u_{2} \in C\left[0, T_{2}\right]$ satisfying Equation (29) and $u_{2}(0)=0 ; u_{3} \in C\left[0, T_{3}\right]$ satisfying Equation (30) and $u_{3}(0)=0 ; u_{i} \in C\left[0, T_{i}\right]$ satisfying Equation (31) and $u_{i}(0)=0\left(i=4,5, \cdots, n^{*}\right)\left(T_{n^{*}}=T\right) ; u_{T} \in C[0,+\infty)$ satisfying Equation (32) and $u_{T}(0)=0$.

The following is the definition of approximate solution of the problem (1).

Definition 5. If there exist $T>0$, natural number $n^{*} \in N$ and intervals $\left[0, T_{1}\right],\left(T_{1}, T_{2}\right], \cdots,\left(T_{n^{*}-1}, T\right]$, $(T,+\infty)$ and functions defined in Equations (13) and (14), such that the problem (26) exists one unique solution, then, we say this solution of the problem (26) is one unique approximate solution of the problem (1).

Our main result is as follows.

Theorem 1. Let conditions $\left(A_{1}\right),\left(A_{2}\right),\left(A_{3}\right)$ hold, then the problem (1) exists one unique approximate solution.

Proof of Theorem 1. From Definitions 4 and 5 and Lemma 6, we only need to consider the unique existence of solution of the problem (26). According to the above analysis, equation of problem (26) can be written as the Equation (27). So Equation (26) in the interval $\left(0, T_{1}\right]$ can be written as (28). Applying operator $I_{0+}^{p_{1}}$ to both sides of (28), by Lemma 5, we have

$$
x(t)=c t^{p_{1}-1}+\frac{1}{\Gamma\left(p_{1}\right)} \int_{0}^{t}(t-s)^{p_{1}-1} f\left(s, x(s), D_{0+}^{q_{1}} x(s)\right) d s, 0<t \leq T_{1} .
$$

By $x(0)=0$ and the assumption of function $f$, we get $c=0$, that is

$$
x(t)=\frac{1}{\Gamma\left(p_{1}\right)} \int_{0}^{t}(t-s)^{p_{1}-1} f\left(s, x(s), D_{0+}^{q_{1}} x(s)\right) d s, 0 \leq t \leq T_{1} .
$$

Let $D_{0+}^{q_{1}} x(t)=y(t)$, then, according to $x(0)=0$ and Lemma 5 , we get that

$$
x(t)=I_{0+}^{q_{1}} y(t),
$$

hence we will consider existence of solution to integral equation as following

$$
y(t)=\frac{1}{\Gamma\left(p_{1}-q_{1}\right)} \int_{0}^{t}(t-s)^{p_{1}-q_{1}-1} f\left(s, I_{0+}^{q_{1}} y(s), y(s)\right) d s, 0 \leq t \leq T_{1} .
$$

Obviously, if $y^{*} \in B_{1}=C\left[0, T_{1}\right]$ is a solution of (34), then, applying operator $I_{0+}^{q_{1}}$ on both sides of (34), from Lemma 2, it holds

$$
I_{0+}^{q_{1}} y^{*}(t)=I_{0+}^{q_{1}} I_{0+}^{p_{1}-q_{1}} f\left(t, I_{0+}^{q_{1}} y^{*}(t), y^{*}(t)\right)=I_{0+}^{p_{1}} f\left(t, I_{0+}^{q_{1}} y^{*}(t), y^{*}(t)\right), 0 \leq t \leq T_{1},
$$

let

$$
I_{0+}^{q_{1}} y^{*}(t)=x^{*}(t), 0 \leq t \leq T
$$

as a result, we have that

$$
x^{*}(t)=I_{0+}^{p_{1}} f\left(t, x^{*}(t), D_{0+}^{q_{1}} x^{*}(t)\right), 0 \leq t \leq T_{1},
$$

that is, $x^{*} \in B_{1}=C\left[0, T_{1}\right]$ is a solution of (33), thus, we know that $x^{*} \in B_{1}=C\left[0, T_{1}\right]$ is a solution of Equation (28) with zero initial value condition. 
Define operator $F: B_{1} \rightarrow B_{1}$ by

$$
F y(t)=\frac{1}{\Gamma\left(p_{1}-q_{1}\right)} \int_{0}^{t}(t-s)^{p_{1}-q_{1}-1} f\left(s, I_{0+}^{q_{1}} y(s), y(s)\right) d s, 0 \leq t \leq T_{1} .
$$

From the continuity of function $f$ and the standard arguments, we know that the operator $F: B_{1} \rightarrow B_{1}$ is well defined. Let $M=\max _{0 \leq t \leq T}|f(t, 0,0)|$. Let $\Omega_{1}$ be a bounded, convex and closed subset of $B_{1}$ defined by

$$
\Omega_{1}=\left\{y\left|y \in B_{1} ;\right| y(t) \mid \leq K_{1} e^{R_{1}^{2} t_{1}^{p_{1}-q_{1}}}, 0 \leq t \leq T_{1}\right\},
$$

where

$$
K_{1}=\frac{2 M T_{1}^{p_{1}-q_{1}}}{\Gamma\left(1+p_{1}-q_{1}\right)}
$$

$R_{1} \in N$ satisfying

$$
R_{1}>\left\{1,\left(\frac{2 d_{1}\left(1+T_{1}^{p_{1}-q_{1}}\right)}{p_{1}-q_{1}}\right)^{\frac{1}{p_{1}-q_{1}}}\right\},
$$

here $d_{1}=\frac{1}{\Gamma\left(p_{1}-q_{1}\right)}\left[\frac{c_{1} T_{1}^{q_{1}}}{\Gamma\left(1+q_{1}\right)}+c_{2}\right]\left(c_{1}, c_{2}\right.$ are the constants appearing in condition $\left.\left(A_{2}\right)\right)$.

By the analogy way as in [23], we could verify that $F: \Omega_{1} \rightarrow \Omega_{1}$ is well defined. In fact, for $y \in \Omega_{1}$, since

$$
\begin{aligned}
\left|I_{0+}^{q_{1}} y(s)\right| & \leq \frac{1}{\Gamma\left(q_{1}\right)} \int_{0}^{s}(s-\tau)^{q_{1}-1}|y(\tau)| d \tau \\
& \leq \frac{K_{1}}{\Gamma\left(q_{1}\right)} \int_{0}^{s}(s-\tau)^{q_{1}-1} e^{R_{1}^{2} \tau^{p_{1}-q_{1}}} d \tau \\
& \leq \frac{K_{1}}{\Gamma\left(q_{1}\right)} \int_{0}^{s}(s-\tau)^{q_{1}-1} e^{R_{1}^{2} s^{p_{1}-q_{1}}} d \tau \\
& =\frac{K_{1}}{\Gamma\left(1+q_{1}\right)} s^{q_{1}} e^{R_{1}^{2} s^{p_{1}-q_{1}}} \\
& \leq \frac{K_{1} T_{1}^{q_{1}}}{\Gamma\left(1+q_{1}\right)} e^{R_{1}^{2} s^{p_{1}-q_{1}}} .
\end{aligned}
$$

Now, $y \in \Omega_{1}$, by estimations above and $\left(A_{2}\right)$, we get

$$
\begin{aligned}
& |F y(t)| \\
\leq & \frac{1}{\Gamma\left(p_{1}-q_{1}\right)} \int_{0}^{t}(t-s)^{p_{1}-q_{1}-1}\left|f\left(s, I_{0+}^{q_{1}} y(s), y(s)\right)\right| d s \\
= & \frac{1}{\Gamma\left(p_{1}-q_{1}\right)} \int_{0}^{t}(t-s)^{p_{1}-q_{1}-1}\left|f\left(s, I_{0+}^{q_{1}} y(s), y(s)\right)-f(s, 0,0)+f(s, 0,0)\right| d s \\
\leq & \frac{M T_{1}^{p_{1}-q_{1}}}{\Gamma\left(1+p_{1}-q_{1}\right)}+\frac{1}{\Gamma\left(p_{1}-q_{1}\right)} \int_{0}^{t}(t-s)^{p_{1}-q_{1}-1}\left(c_{1} \frac{\left|I_{0+}^{q_{1}} y(s)\right|}{1+s^{\lambda}}+c_{2} \frac{|y(s)|}{1+s^{\lambda}}\right) d s \\
\leq & \frac{K_{1}}{2}+\frac{1}{\Gamma\left(p_{1}-q_{1}\right)} \int_{0}^{t}(t-s)^{p_{1}-q_{1}-1}\left(c_{1}\left|I_{0+}^{q_{1}} y(s)\right|+c_{2}|y(s)|\right) d s
\end{aligned}
$$




$$
\begin{aligned}
& \leq \quad \frac{K_{1}}{2}+\frac{1}{\Gamma\left(p_{1}-q_{1}\right)} \int_{0}^{t}(t-s)^{p_{1}-q_{1}-1}\left(\frac{K_{1} c_{1} T_{1}^{q_{1}}}{\Gamma\left(1+q_{1}\right)} e^{R_{1}^{2} s^{p_{1}-q_{1}}}+c_{2} K_{1} e^{R_{1}^{2} s^{p_{1}-q_{1}}}\right) d s \\
& \leq \frac{K_{1}}{2}+K_{1} d_{1}\left[\sum_{i=1}^{R_{1}-1} \int_{\frac{(i-1) t}{R_{1}}}^{\frac{i t}{R_{1}}}(t-s)^{p_{1}-q_{1}-1} e^{R_{1}^{2} s p_{1}-q_{1}} d s\right. \\
& \left.+\int_{\frac{\left(R_{1}-1\right) t}{R_{1}}}^{t}(t-s)^{p_{1}-q_{1}-1} e^{R_{1}^{2} s^{p_{1}-q_{1}}} d s\right] \\
& \leq \frac{K_{1}}{2}+K_{1} d_{1}\left[\sum_{i=1}^{R_{1}-1} \int_{\frac{(i-1) t}{R_{1}}}^{\frac{i t}{R_{1}}} R_{1}^{1-p_{1}+q_{1}}\left(R_{1}-i\right)^{p_{1}-q_{1}-1} t^{p_{1}-q_{1}-1} e^{R_{1}^{2} s^{p_{1}-q_{1}}} d s\right. \\
& \left.+\int_{\frac{\left(R_{1}-1\right) t}{R_{1}}}^{t}(t-s)^{p_{1}-q_{1}-1} e^{R_{1}^{2} t^{p_{1}-q_{1}}} d s\right] \\
& \leq \quad \frac{K_{1}}{2}+K_{1} d_{1}\left[\sum_{i=1}^{R_{1}-1} \int_{\frac{(i-1) t}{R_{1}}}^{\frac{i t}{R_{1}}} R_{1}^{1-p_{1}+q_{1}} t^{p_{1}-q_{1}-1} e^{R_{1}^{2} s^{p_{1}-q_{1}}} d s\right. \\
& \left.+\int_{\frac{\left(R_{1}-1\right) t}{R_{1}}}^{t}(t-s)^{p_{1}-q_{1}-1} e^{R_{1}^{2} p^{p_{1}-q_{1}}} d s\right] \\
& =\frac{K_{1}}{2}+K_{1} d_{1} R_{1}^{1-p_{1}+q_{1}} \int_{0}^{\frac{\left(R_{1}-1\right) t}{R_{1}}} t^{p_{1}-q_{1}-1} e^{R_{1}^{2} p_{1}-q_{1}} d s+\frac{K_{1} d_{1} R_{1}^{q_{1}-p_{1}} T_{1}^{p_{1}-q_{1}}}{p_{1}-q_{1}} e^{R_{1}^{2} t p_{1}-q_{1}} \\
& \leq \quad \frac{K_{1}}{2}+K_{1} d_{1} R_{1}^{1-p_{1}+q_{1}} \int_{0}^{\frac{\left(R_{1}-1\right) t}{R_{1}}} s^{p_{1}-q_{1}-1} e^{R_{1}^{2} s^{p_{1}-q_{1}}} d s+\frac{K_{1} d_{1} R_{1}^{q_{1}-p_{1}} T_{1}^{p_{1}-q_{1}}}{p_{1}-q_{1}} e^{R_{1}^{2} p_{1}-q_{1}} \\
& \leq \quad \frac{K_{1}}{2}+\frac{K_{1} d_{1} R_{1}^{1-p_{1}+q_{1}}}{R_{1}^{2}\left(p_{1}-q_{1}\right)} e^{R_{1}^{2}\left(\frac{\left(R_{1}-1\right) t}{R_{1}}\right)^{p_{1}-q_{1}}}+\frac{K_{1} d_{1} R_{1}^{q_{1}-p_{1}} T_{1}^{p_{1}-q_{1}}}{p_{1}-q_{1}} e^{R_{1}^{2} t^{p_{1}-q_{1}}} \\
& \leq \quad \frac{K_{1}}{2}+\frac{K_{1} d_{1} R_{1}^{-1-p_{1}+q_{1}}}{p_{1}-q_{1}} e^{R_{1}^{2} t_{1}-q_{1}}+\frac{K_{1} d_{1} R_{1}^{q_{1}-p_{1}} T_{1}^{p_{1}-q_{1}}}{p_{1}-q_{1}} e^{R_{1}^{2} t p_{1}-q_{1}} \\
& \leq \quad \frac{K_{1}}{2} e^{R_{1}^{2} p_{1}-q_{1}}+\frac{K_{1} d_{1}\left(1+T_{1}^{p_{1}-q_{1}}\right)}{p_{1}-q_{1}} R_{1}^{q_{1}-p_{1}} e^{R_{1}^{2} p_{1}-q_{1}} \\
& \leq \quad \frac{K_{1}}{2} e^{R_{1}^{2} t p_{1}-q_{1}}+\frac{K_{1}}{2} e^{R_{1}^{2} t_{1}^{p_{1}}-q_{1}}=K_{1} e^{R_{1}^{2} p_{1}-q_{1}},
\end{aligned}
$$

which implies that $F: \Omega_{1} \rightarrow \Omega_{1}$ is well defined. By the standard arguments, we could know that $F: \Omega_{1} \rightarrow \Omega_{1}$ is a completely operator. Hence, the Schauder fixed point theorem assures that operator $F$ has at least one fixed point $y_{1}(t) \in \Omega_{1}$. Obviously, $y_{1}(0)=0$. Now, we will verify the uniqueness of solution to the integral Equation (34). We notice that: for $0 \leq s \leq t \leq T_{1}$, if $0 \leq t-s \leq 1$, then $(t-s)^{p_{1}-1} \leq(t-s)^{p_{1}-q_{1}-1}$; if $t-s \geq 1$, then $(t-s)^{p_{1}-q_{1}-1} \leq(t-s)^{p_{1}-1}$. As a result, we take

$$
\max \left\{(t-s)^{p_{1}-1},(t-s)^{p_{1}-q_{1}-1}\right\} \doteq(t-s)^{\alpha-1},
$$

where $\alpha$ denotes $p_{1}$ or $p_{1}-q_{1}$. Now, let $u_{1}(t), u_{2}(t)$ zre two solutions of the integral Equation (34), by expression above and $\left(A_{2}\right)$, we get

$$
\begin{aligned}
& \left|u_{1}(t)-u_{2}(t)\right| \\
\leq & \frac{1}{\Gamma\left(p_{1}-q_{1}\right)} \int_{0}^{t}(t-s)^{p_{1}-q_{1}-1}\left(c_{1} \frac{\left|I_{0+}^{q_{1}}\left(u_{1}(s)-u_{2}(s)\right)\right|}{1+s^{\lambda}}+c_{2} \frac{\left|u_{1}(s)-u_{2}(s)\right|}{1+s^{\lambda}}\right) d s
\end{aligned}
$$




$$
\begin{aligned}
\leq \quad & \frac{1}{\Gamma\left(p_{1}-q_{1}\right)} \int_{0}^{t}(t-s)^{p_{1}-q_{1}-1}\left(c_{1}\left|I_{0+}^{q_{1}}\left(u_{1}(s)-u_{2}(s)\right)\right|+c_{2}\left|u_{1}(s)-u_{2}(s)\right|\right) d s \\
\leq \quad & \frac{c_{1}}{\Gamma\left(p_{1}-q_{1}\right) \Gamma\left(q_{1}\right)} \int_{0}^{t}(t-s)^{p_{1}-q_{1}-1} \int_{0}^{s}(s-\tau)^{q_{1}-1}\left|u_{1}(\tau)-u_{2}(\tau)\right| d \tau d s \\
& +\frac{c_{2}}{\Gamma\left(p_{1}-q_{1}\right)} \int_{0}^{t}(t-s)^{p_{1}-q_{1}-1}\left|u_{1}(s)-u_{2}(s)\right| d s \\
=\quad & \frac{c_{1}}{\Gamma\left(p_{1}-q_{1}\right) \Gamma\left(q_{1}\right)} \int_{0}^{t} \int_{\tau}^{t}(t-s)^{p_{1}-q_{1}-1}(s-\tau)^{q_{1}-1}\left|u_{1}(\tau)-u_{2}(\tau)\right| d s d \tau \\
= & +\frac{c_{2}}{\Gamma\left(p_{1}-q_{1}\right)} \int_{0}^{t}(t-s)^{p_{1}-q_{1}-1}\left|u_{1}(s)-u_{2}(s)\right| d s \\
= & \frac{c_{1}}{\Gamma\left(p_{1}\right)} \int_{0}^{t}(t-\tau)^{p_{1}-1}\left|u_{1}(\tau)-u_{2}(\tau)\right| d \tau+\frac{c_{2}}{\Gamma\left(p_{1}-q_{1}\right)} \int_{0}^{t}(t-s)^{p_{1}-q_{1}-1}\left|u_{1}(s)-u_{2}(s)\right| d s \\
\leq \quad & \frac{c_{1}}{\Gamma\left(p_{1}\right)} \int_{0}^{t}(t-\tau)^{\alpha-1}\left|u_{1}(\tau)-u_{2}(\tau)\right| d \tau+\frac{c_{2}}{\Gamma\left(p_{1}-q_{1}\right)} \int_{0}^{t}(t-s)^{\alpha-1}\left|u_{1}(s)-u_{2}(s)\right| d s \\
=\quad & \left.\frac{c_{1}}{\Gamma\left(p_{1}\right)}+\frac{c_{2}}{\Gamma\left(p_{1}-q_{1}\right)}\right] \int_{0}^{t}(t-\tau)^{\alpha-1}\left|u_{1}(\tau)-u_{2}(\tau)\right| d \tau,
\end{aligned}
$$

by Lemma 7, we obtain that $u_{1}(t)=u_{2}(t), 0 \leq t \leq T_{1}$, this assures the uniqueness of solution of (34). As a result, by some arguments above, $x_{1}(t)=I_{0+}^{q_{1}} y_{1}(t)$ is one unique solution of the Equation (28) with zero initial value condition.

Also, we have obtained that the Equation (27) in the interval $\left(T_{1}, T_{2}\right]$ can be written by (29). In order to consider the existence result of solutions to (29), we may discuss the following equation defined on interval $\left(0, T_{2}\right]$

$$
\frac{d}{d t} \int_{0}^{t} \frac{(t-s)^{-p_{2}} x(s)}{\Gamma\left(1-p_{2}\right)} d s=D_{0+}^{p_{2}} x(t)=f\left(t, x, \frac{d}{d t} \int_{0}^{t} \frac{(t-s)^{-q_{2}} x(s)}{\Gamma\left(1-q_{2}\right)} d s\right)=f\left(t, x, D_{0+}^{q_{2}} x\right) .
$$

It is clear that if function $x \in C\left[0, T_{2}\right]$ satisfies the Equation (36), then $x(t)$ must satisfy the Equation (29). In fact, if $x^{*} \in C\left[0, T_{2}\right]$ with $x^{*}(0)=0$ is a solution of the Equation (36) with initial value condition $x(0)=0$, that is

$$
\begin{aligned}
& D_{0+}^{p_{2}} x^{*}(t) \\
= & \frac{d}{d t} \int_{0}^{t} \frac{(t-s)^{-p_{2}} x^{*}(s)}{\Gamma\left(1-p_{2}\right)} d s \\
= & f\left(t, x^{*}(t), D_{0+}^{q_{2}} x^{*}(t)\right)=f\left(t, x^{*}(t), \frac{d}{d t} \int_{0}^{t} \frac{(t-s)^{-q_{2}} x^{*}(s)}{\Gamma\left(1-q_{2}\right)} d s\right), 0<t \leq T_{2} ; x^{*}(0)=0 .
\end{aligned}
$$

Hence, from the equality above, we have that $x^{*} \in C\left[0, T_{2}\right]$ with $x^{*}(0)=0$ satisfies the equation

$$
\frac{d}{d t} \int_{0}^{t} \frac{(t-s)^{-p_{2}} x^{*}(s)}{\Gamma\left(1-p_{2}\right)} d s=f\left(t, x^{*}(t), \frac{d}{d t} \int_{0}^{t} \frac{(t-s)^{-q_{2}} x^{*}(s)}{\Gamma\left(1-q_{2}\right)} d s\right), \quad T_{1} \leq t \leq T_{2},
$$

which means the function $x^{*} \in C\left[0, T_{2}\right]$ with $x^{*}(0)=0$ is a solution of the Equation (29).

Based on this fact, we consider the existence of solutions to the Equation (36) with initial value condition $x(0)=0$. 
Now, applying operator $I_{0+}^{p_{2}}$ on both sides of (36), by Lemma 5, we have

$$
x(t)=c t^{p_{2}-1}+\frac{1}{\Gamma\left(p_{2}\right)} \int_{0}^{t}(t-s)^{p_{2}-1} f\left(s, x(s), D_{0+}^{q_{2}} x(s)\right) d s, 0<t \leq T_{2} .
$$

By initial value condition $x(0)=0$, we have $c=0$, that is

$$
x(t)=\frac{1}{\Gamma\left(p_{2}\right)} \int_{0}^{t}(t-s)^{p_{2}-1} f\left(s, x(s), D_{0+}^{q_{2}} x(s)\right) d s, 0 \leq t \leq T_{2} .
$$

Let $D_{0+}^{q_{2}} x(t)=y(t)$, then, according to $x(0)=0$ and Lemma 5, we get that

$$
x(t)=I_{0+}^{q_{2}} y(t),
$$

hence we will consider existence of solution to integral equation as following

$$
y(t)=\frac{1}{\Gamma\left(p_{2}-q_{2}\right)} \int_{0}^{t}(t-s)^{p_{2}-q_{2}-1} f\left(s, I_{0+}^{q_{2}} y(s), y(s)\right) d s, 0 \leq t \leq T_{2} .
$$

Obviously, if $y^{*} \in B_{2}=C\left[0, T_{2}\right]$ is a solution of (38), then, by (38) and Lemma 2, it holds

$$
I_{0+}^{q_{2}} y^{*}(t)=I_{0+}^{q_{2}} I_{0+}^{p_{2}-q_{2}} f\left(t, I_{0+}^{q_{2}} y^{*}(t), y^{*}(t)\right)=I_{0+}^{p_{2}} f\left(t, I_{0+}^{q_{2}} y^{*}(t), y^{*}(t)\right), 0 \leq t \leq T_{2}
$$

let

$$
I_{0+}^{q_{2}} y^{*}(t)=x^{*}(t), 0 \leq t \leq T_{2}
$$

as a result, we have that

$$
x^{*}(t)=I_{0+}^{p_{2}} f\left(t, x^{*}(t), D_{0+}^{q_{2}} x^{*}(t)\right), 0 \leq t \leq T_{2},
$$

that is, $x^{*} \in B_{2}=C\left[0, T_{2}\right]$ is a solution of (37), hence, $x^{*} \in B_{2}=C\left[0, T_{2}\right]$ is a solution of Equation (29) with zero initial value condition.

Define operator $F: B_{2} \rightarrow B_{2}$ by

$$
F y(t)=\frac{1}{\Gamma\left(p_{2}-q_{2}\right)} \int_{0}^{t}(t-s)^{p_{2}-q_{2}-1} f\left(s, I_{0+}^{q_{2}} y(s), y(s)\right) d s, \quad 0 \leq t \leq T_{2} .
$$

From the continuity of function $f$ and the standard arguments, we know that the operator $F: B_{1} \rightarrow B_{2}$ is well defined. Let $\Omega_{2}$ be a bounded, convex and closed subset of $B_{2}$ defined by

$$
\Omega_{2}=\left\{y\left|y \in B_{2} ;\right| y(t) \mid \leq K_{2} e^{R_{2}^{2} t_{2}-q_{2}}, 0 \leq t \leq T_{2}\right\},
$$

where

$$
K_{2}=\frac{2 M T_{2}^{p_{2}-q_{2}}}{\Gamma\left(1+p_{2}-q_{2}\right)}
$$

$R_{2} \in N$ satisfying

$$
R_{2}>\left\{1,\left(\frac{2 d_{2}\left(1+T_{2}^{p_{2}-q_{2}}\right)}{p_{2}-q_{2}}\right)^{\frac{1}{p_{2}-q_{2}}}\right\}
$$

here $d_{2}=\frac{1}{\Gamma\left(p_{2}-q_{2}\right)}\left[\frac{c_{1} T_{2}^{q_{2}}}{\Gamma\left(1+q_{2}\right)}+c_{2}\right]\left(c_{1}, c_{2}\right.$ are the constants appearing in condition $\left.\left(A_{2}\right)\right)$. By the same arguments above, there exists $y_{2} \in \Omega_{2}$ such that $x_{2}(t)=I_{0+}^{q_{2}} y_{2}(t)$ is one unique solution of the Equation (29) with zero initial value condition. 
In a similar way, for $i=3, \cdots, n^{*}$, we get that the Equation (31) defined on $\left(T_{i-1}, T_{i}\right]\left(T_{n^{*}}=T\right)$ has one solution $x_{i}(t) \in \Omega_{i} \subset B_{i}$ with $x_{i}(0)=0$, where

$$
\begin{gathered}
\Omega_{i}=\left\{y\left|y \in B_{i} ;\right| y(t) \mid \leq K_{i} e^{R_{i}^{2} p_{i}-q_{i}}, 0 \leq t \leq T_{i}\right\}, \\
K_{i}=\frac{2 M T_{i}^{p_{i}-q_{i}}}{\Gamma\left(1+p_{i}-q_{i}\right)},
\end{gathered}
$$

$R_{i} \in N$ satisfying

$$
R_{i}>\left\{1,\left(\frac{2 d_{i}\left(1+T_{i}^{p_{i}-q_{i}}\right)}{p_{i}-q_{i}}\right)^{\frac{1}{p_{i}-q_{i}}}\right\}
$$

here $d_{i}=\frac{1}{\Gamma\left(p_{i}-q_{i}\right)}\left[\frac{c_{1} q_{i}^{q_{i}}}{\Gamma\left(1+q_{i}\right)}+c_{2}\right]\left(c_{1}, c_{2}\right.$ are the constants appearing in condition $\left.\left(A_{2}\right)\right), i=3,4, \cdots, n^{*}$, $T_{n^{*}}=T$.

Finally, we get that the Equation (27) in the interval $(T,+\infty)$ can be written by (32). In order to consider the existence result of solutions to (32), we may discuss the following equation defined on interval $(0,+\infty)$

$$
\frac{d}{d t} \int_{0}^{t} \frac{(t-s)^{-\rho_{1}}}{\Gamma\left(1-\rho_{1}\right)} x(s) d s=D_{0+}^{\rho_{1}} x(t)=f\left(t, x, D_{0+}^{\rho_{2}} x\right), 0<t<+\infty .
$$

We see that, if function $x \in C[0,+\infty)$ satisfies the Equation (39), then $x(t)$ must satisfy the Equation (32). In fact, if $x^{*} \in C[0,+\infty)$ with $x^{*}(0)=0$ is a solution of the Equation (39) with initial value condition $x(0)=0$, that is

$$
\begin{aligned}
D_{0+}^{\rho_{1}} x^{*}(t) & =\frac{d}{d t} \int_{0}^{t} \frac{(t-s)^{-\rho_{1}} x^{*}(s)}{\Gamma\left(1-\rho_{1}\right)} d s=f\left(t, x^{*}(t), D_{0+}^{\rho_{2}} x^{*}\right) \\
& =f\left(t, x^{*}(t), \frac{d}{d t} \int_{0}^{t} \frac{(t-s)^{-\rho_{2}} x^{*}(s)}{\Gamma\left(1-\rho_{2}\right)} d s\right), 0<t<+\infty ; x^{*}(0)=0 .
\end{aligned}
$$

Hence, from the equality above, we have $x^{*} \in C[0,+\infty)$ with $x^{*}(0)=0$ satisfying the equation

$$
\frac{d}{d t} \int_{0}^{t} \frac{(t-s)^{-\rho_{1}} x(s)}{\Gamma\left(1-\rho_{1}\right)} d s=f\left(t, x(t), \frac{d}{d t} \int_{0}^{t} \frac{(t-s)^{-\rho_{2}} x(s)}{\Gamma\left(1-\rho_{2}\right)} d s\right), \quad T<t<+\infty,
$$

which means the function $x^{*} \in C[0,+\infty)$ with $x^{*}(0)=0$ is a solution of the Equation (32).

Based on this fact, we will consider the existence of solutions to the Equation (39) with initial value condition $x(0)=0$.

Now, applying operator $I_{0+}^{\rho_{1}}$ on both sides of (39), by Lemma 5, we have that

$$
x(t)=c t^{\rho_{1}-1}+\frac{1}{\Gamma\left(\rho_{1}\right)} \int_{0}^{t}(t-s)^{\rho_{1}-1} f\left(s, x(s), D_{0+}^{\rho_{2}} x(s)\right) d s, 0<t<+\infty .
$$

By initial value condition $x(0)=0$, we have $c=0$, that is

$$
x(t)=\frac{1}{\Gamma\left(\rho_{1}\right)} \int_{0}^{t}(t-s)^{\rho_{1}-1} f\left(s, x(s), D_{0+}^{\rho_{2}} x(s)\right) d s, 0 \leq t<+\infty .
$$

Similar to arguments above, we let $D_{0+}^{\rho_{2}} x(t)=y(t)$, then, according to $x(0)=0$ and Lemma 5, we get that

$$
x(t)=I_{0+}^{\rho_{2}} y(t),
$$


hence we will consider existence of solution to integral equation as following

$$
y(t)=\frac{1}{\Gamma\left(\rho_{1}-\rho_{2}\right)} \int_{0}^{t}(t-s)^{\rho_{1}-\rho_{2}-1} f\left(s, I_{0+}^{\rho_{2}} y(s), y(s)\right) d s, 0 \leq t<+\infty .
$$

Obviously, if $y^{*} \in E$ is a solution of (41), then, by (41) and Lemma 2, it holds

$$
I_{0+}^{\rho_{2}} y^{*}(t)=I_{0+}^{\rho_{2}} I_{0+}^{\rho_{1}-\rho_{2}} f\left(t, I_{0+}^{\rho_{2}} y^{*}(t), y^{*}(t)\right)=I_{0+}^{\rho_{1}} f\left(t, I_{0+}^{\rho_{2}} y^{*}(t), y^{*}(t)\right), 0 \leq t<+\infty .
$$

Let

$$
I_{0+}^{\rho_{2}} y^{*}(t)=x^{*}(t), 0 \leq t<+\infty .
$$

As a result, we have that

$$
x^{*}(t)=I_{0+}^{\rho_{1}} f\left(t, x^{*}(t), D_{0+}^{\rho_{2}} x^{*}(t)\right), 0 \leq t<+\infty,
$$

that is, $x^{*} \in E$ is a solution of (40), hence, $x^{*} \in E$ is a solution of Equation (32) with zero initial value condition.

Defining operator $F: E \rightarrow E$ as follows

$$
F y(t)=\frac{1}{\Gamma\left(\rho_{1}-\rho_{2}\right)} \int_{0}^{t}(t-s)^{\rho_{1}-\rho_{2}-1} f\left(s, I_{0+}^{\rho_{2}} y(s), y(s)\right) d s, 0 \leq t<+\infty .
$$

To get the operator $F: E \rightarrow E$ is well defined. First, we verify that $F y \in C[0,+\infty)$ for $x \in E$. In fact, for the case of $t_{0} \in(0,+\infty)$, take $t>t_{0}, t-t_{0}<1$, then

$$
\left(t_{0}-s\right)^{\rho_{1}-1}>(t-s)^{\rho_{1}-1}, 0 \leq s<t_{0} .
$$

Now, for $y \in E$, it holds

$$
\begin{aligned}
\frac{\left|I_{0+}^{\rho_{2}} y(s)\right|}{1+s^{\lambda}} & \leq \frac{\int_{0}^{s}(s-\tau)^{\rho_{2}-1}|y(\tau)| d \tau}{\Gamma\left(\rho_{2}\right)\left(1+s^{\lambda}\right)} \\
& \leq \frac{\int_{0}^{s}(s-\tau)^{\rho_{2}-1}\left(1+\tau^{\lambda}\right)\|y\|_{E} d \tau}{\Gamma\left(\rho_{2}\right)\left(1+s^{\lambda}\right)} \\
& \leq \frac{\int_{0}^{s}(s-\tau)^{\rho_{2}-1}\left(1+s^{\lambda}\right)\|y\|_{E} d \tau}{\Gamma\left(\rho_{2}\right)\left(1+s^{\lambda}\right)} \\
& =\frac{\|y\|_{E^{s}}{ }^{2}}{\Gamma\left(1+\rho_{2}\right)}
\end{aligned}
$$

thus, for $y \in E$, we have

$$
\begin{aligned}
|F y(t)| \leq \quad & \frac{1}{\Gamma\left(\rho_{1}-\rho_{2}\right)} \int_{0}^{t_{0}}\left(\left(t_{0}-s\right)^{\rho_{1}-\rho_{2}-1}-(t-s)^{\rho_{1}-\rho_{2}-1}\right)\left(c_{1} \frac{\left|I_{0+}^{\rho_{2}} y(s)\right|}{1+s^{\lambda}}+c_{2} \frac{|y(s)|}{1+s^{\lambda}}\right) d s \\
& +\frac{1}{\Gamma\left(\rho_{1}-\rho_{2}\right)} \int_{t_{0}}^{t}(t-s)^{\rho_{1}-\rho_{2}-1}\left(c_{1} \frac{\left|I_{0+}^{\rho_{2}} y(s)\right|}{1+s^{\lambda}}+c_{2} \frac{|y(s)|}{1+s^{\lambda}}\right) d s \\
& +\frac{1}{\Gamma\left(\rho_{1}-\rho_{2}\right)} \int_{0}^{t_{0}}\left(\left(t_{0}-s\right)^{\rho_{1}-\rho_{2}-1}-(t-s)^{\rho_{1}-\rho_{2}-1}\right)|f(s, 0,0)| d s \\
& +\frac{1}{\Gamma\left(\rho_{1}-\rho_{2}\right)} \int_{t_{0}}^{t}(t-s)^{\rho_{1}-\rho_{2}-1}|f(s, 0,0)| d s
\end{aligned}
$$




$$
\begin{aligned}
\leq \quad & \frac{\|y\|_{E}}{\Gamma\left(\rho_{1}-\rho_{2}\right)} \int_{0}^{t_{0}}\left(\left(t_{0}-s\right)^{\rho_{1}-\rho_{2}-1}-(t-s)^{\rho_{1}-\rho_{2}-1}\right)\left(c_{1} \frac{s^{\rho_{2}}}{\Gamma\left(1+\rho_{2}\right)}+c_{2}\right) d s \\
& +\frac{\|y\|_{E}}{\Gamma\left(\rho_{1}-\rho_{2}\right)} \int_{t_{0}}^{t}(t-s)^{\rho_{1}-\rho_{2}-1}\left(c_{1} \frac{s^{\rho_{2}}}{\Gamma\left(1+\rho_{2}\right)}+c_{2}\right) d s \\
& +\frac{\max _{0 \leq t \leq t_{0}+1}|f(t, 0,0)|}{\Gamma\left(\rho_{1}-\rho_{2}\right)} \int_{0}^{t_{0}}\left(\left(t_{0}-s\right)^{\rho_{1}-\rho_{2}-1}-(t-s)^{\rho_{1}-\rho_{2}-1}\right) d s \\
& +\frac{\max _{0 \leq t \leq t_{0}+1}|f(t, 0,0)|}{\Gamma\left(\rho_{1}-\rho_{2}\right)} \int_{t_{0}}^{t}(t-s)^{\rho_{1}-\rho_{2}-1} d s .
\end{aligned}
$$

We will consider the four terms above, respectively. For $0<\eta<\rho_{1}-\rho_{2}$, it is easy to show that

$$
\int_{0}^{t}(t-s)^{\rho_{1}-\rho_{2}-1} s^{\eta} d s=\frac{\Gamma(1+\eta) \Gamma\left(\rho_{1}-\rho_{2}\right) t^{\rho_{1}-\rho_{2}+\eta}}{\Gamma\left(1+\rho_{1}-\rho_{2}+\eta\right)} .
$$

Hence, for any given $\varepsilon>0$, there exists a $\delta_{1}>0$, such that, when $0 \leq t_{0} \leq \delta_{1}$, it holds that

$$
\frac{c_{1}\|y\|_{E}}{\Gamma\left(\rho_{1}-\rho_{2}\right) \Gamma\left(1+\rho_{2}\right)} \int_{0}^{t_{0}}\left(t_{0}-s\right)^{\rho_{1}-\rho_{2}-1} s^{\rho_{2}} d s<\frac{\varepsilon}{4}, \frac{c_{2}\|y\|_{E}}{\Gamma\left(\rho_{2}-\rho_{2}\right)} \int_{0}^{t_{0}}\left(t_{0}-s\right)^{\rho_{1}-\rho_{2}-1} d s<\frac{\varepsilon}{4} .
$$

Moreover, we get

$$
\begin{aligned}
& \int_{\delta_{1}}^{t_{0}}\left(\left(t_{0}-s\right)^{\rho_{1}-\rho_{2}-1}-(t-s)^{\rho_{1}-\rho_{2}-1}\right) s^{\rho_{2}} d s \\
& \leq \quad t_{0}^{\rho_{2}} \int_{\delta_{1}}^{t_{0}}\left(\left(t_{0}-s\right)^{\rho_{1}-\rho_{2}-1}-(t-s)^{\rho_{1}-\rho_{2}-1}\right) d s \\
&= \frac{t_{0}^{\rho_{2}}}{\rho_{1}-\rho_{2}}\left(\left(t_{0}-\delta_{1}\right)^{\rho_{1}-\rho_{2}}-\left(t-\delta_{1}\right)^{\rho_{1}-\rho_{2}}+\left(t-t_{0}\right)^{\rho_{1}-\rho_{2}}\right) \\
& \leq \frac{t_{0}^{\rho_{2}}}{\rho_{1}-\rho_{2}}\left(t-t_{0}\right)^{\rho_{1}-\rho_{2}}, \\
& \int_{\delta_{1}}^{t_{0}}\left(\left(t_{0}-s\right)^{\rho_{1}-\rho_{2}-1}-(t-s)^{\rho_{1}-\rho_{2}-1}\right) d s \leq \frac{1}{\rho_{1}-\rho_{2}}\left(t-t_{0}\right)^{\rho_{1}-\rho_{2}},
\end{aligned}
$$

hence, we know that there exists $\delta_{2}>0$ such that for $0<t-t_{0}<\delta_{2}$, we have

$$
\begin{gathered}
\frac{c_{1}\|y\|_{E}}{\Gamma\left(\rho_{1}-\rho_{2}\right) \Gamma\left(1+\rho_{2}\right)} \int_{\delta_{1}}^{t_{0}}\left(\left(t_{0}-s\right)^{\rho_{1}-\rho_{2}-1}-(t-s)^{\rho_{1}-\rho_{2}-1}\right) s^{\rho_{2}} d s<\frac{\varepsilon}{4}, \\
\frac{c_{2}\|y\|_{E}}{\Gamma\left(\rho_{2}-\rho_{2}\right)} \int_{\delta_{1}}^{t_{0}}\left(\left(t_{0}-s\right)^{\rho_{1}-\rho_{2}-1}-(t-s)^{\rho_{1}-\rho_{2}-1}\right) d s<\frac{\varepsilon}{4},
\end{gathered}
$$

together with (42), it leads to

$$
\int_{0}^{t_{0}}\left(\left(t_{0}-s\right)^{\rho_{1}-\rho_{2}-1}-(t-s)^{\rho_{1}-\rho_{2}-1}\right)\left(\frac{c_{1}\|y\|_{E}{ }^{\rho_{2}}}{\Gamma\left(\rho_{1}-\rho_{2}\right) \Gamma\left(1+\rho_{2}\right)}+\frac{c_{2}\|y\|_{E}}{\Gamma\left(\rho_{2}-\rho_{2}\right)}\right) d s<\varepsilon .
$$

By the direct calculation, we have

$$
\int_{t_{0}}^{t}(t-s)^{\rho_{1}-\rho_{2}-1} s^{\rho_{2}} d s \leq\left(t_{0}+1\right)^{\rho_{2}} \frac{\left(t-t_{0}\right)^{\rho_{1}-\rho_{2}}}{\rho_{1}-\rho_{2}},
$$




$$
\int_{t_{0}}^{t}(t-s)^{\rho_{1}-\rho_{2}-1} d s \leq \frac{\left(t-t_{0}\right)^{\rho_{1}-\rho_{2}}}{\rho_{1}-\rho_{2}}
$$

which implies that there exists $\delta_{3}>0$ such that for $0<t-t_{0}<\delta_{3}$, we get

$$
\int_{t_{0}}^{t}(t-s)^{\rho_{1}-\rho_{2}-1}\left(\frac{c_{1}\|y\|_{E} s^{\rho_{2}}}{\Gamma\left(\rho_{1}-\rho_{2}\right) \Gamma\left(1+\rho_{2}\right)}+\frac{c_{2}\|y\|_{E}}{\Gamma\left(\rho_{2}-\rho_{2}\right)}\right) d s<\varepsilon .
$$

By the same arguments, we get that these estimations still hold for the last two terms above. Hence, we obtain $F x(t)$ is continuous on point $t_{0}$. In view of the arbitrariness of $t_{0}$, we have $F x \in C(0,+\infty)$.

For the case of $t_{0}=0$, by $\left(A_{2}\right)$, for $y \in E$, take $t<1$, then

$$
\begin{aligned}
|F y(t)|= & \left|\frac{1}{\Gamma\left(\rho_{1}-\rho_{2}\right)} \int_{0}^{t}(t-s)^{\rho_{1}-\rho_{2}-1} f\left(s, I_{0+}^{\rho_{2}} y(s), y(s)\right) d s\right| \\
\leq \quad & \left.\frac{\|y\|_{E}}{\Gamma\left(\rho_{1}-\rho_{2}\right)} \int_{0}^{t}(t-s)^{\rho_{1}-\rho_{2}-1}\right)\left(c_{1} \frac{s^{\rho_{2}}}{\Gamma\left(1+\rho_{2}\right)}+c_{2}\right) d s \\
& +\frac{\max _{0 \leq t \leq 1}|f(t, 0,0)|}{\Gamma\left(\rho_{1}-\rho_{2}\right)} \int_{0}^{t}(t-s)^{\rho_{1}-\rho_{2}-1} d s
\end{aligned}
$$

From the previous arguments, we could know that $F y(t)$ is continuous on point 0 . As a result, we have $F y \in C[0,+\infty)$ for $x \in E$.

By the similar arguments, for $y \in E$, by $\left(A_{2}\right)$, we have

$$
\begin{aligned}
\left|\frac{F y(t)}{1+t^{\lambda}}\right| \leq \quad & \frac{1}{\Gamma\left(\rho_{1}-\rho_{2}\right)\left(1+t^{\lambda}\right)} \int_{0}^{t}(t-s)^{\rho_{1}-\rho_{2}-1}\left(c_{1} \frac{\left|I_{0+}^{\rho_{2}} y(s)\right|}{1+s^{\lambda}}+c_{2} \frac{|y(s)|}{1+s^{\lambda}}\right) d s \\
& +\frac{1}{\Gamma\left(\rho_{1}-\rho_{2}\right)\left(1+t^{\lambda}\right)} \int_{0}^{t}(t-s)^{\rho_{1}-\rho_{2}-1}|f(s, 0,0)| d s \\
\leq \quad & \frac{\|y\|_{E}}{\Gamma\left(\rho_{1}-\rho_{2}\right)\left(1+t^{\lambda}\right)} \int_{0}^{t}(t-s)^{\rho_{1}-\rho_{2}-1}\left(c_{1} \frac{s^{\rho_{2}}}{\Gamma\left(1+\rho_{2}\right)}+c_{2}\right) d s \\
& +\frac{1}{\Gamma\left(\rho_{1}-\rho_{2}\right)\left(1+t^{\lambda}\right)} \int_{0}^{t}(t-s)^{\rho_{1}-\rho_{2}-1}|f(s, 0,0)| d s \\
=\quad & \frac{\|y\|_{E}}{1+t^{\lambda}}\left[\frac{c_{1} t^{\rho_{1}}}{\Gamma\left(1+\rho_{1}\right)}+\frac{c_{2} t^{\rho_{1}-\rho_{2}}}{\Gamma\left(1+\rho_{1}-\rho_{2}\right)}\right] \\
& +\frac{1}{\Gamma\left(\rho_{1}-\rho_{2}\right)\left(1+t^{\lambda}\right)} \int_{0}^{t}(t-s)^{\rho_{1}-\rho_{2}-1}|f(s, 0,0)| d s,
\end{aligned}
$$

according to these estimations and $\left(A_{2}\right)$, we ge that $\lim _{t \rightarrow+\infty} \frac{F y(t)}{1+t^{\lambda}}=0$. Hence, $F: E \rightarrow E$ is well defined.

Now, for $x, y \in E$, by a similar way, we get

$$
\begin{aligned}
& \frac{|F x(t)-F y(t)|}{1+t^{\lambda}} \\
\leq & \frac{1}{\Gamma\left(\rho_{1}-\rho_{2}\right)} \int_{0}^{t}(t-s)^{\rho_{1}-\rho_{2}-1}\left(c_{1} \frac{I_{0+}^{\rho_{2}}|x(s)-y(s)|}{1+s^{\lambda}}+c_{2} \frac{|x(s)-y(s)|}{1+s^{\lambda}}\right) d s \\
\leq & \frac{\|x-y\|_{E}}{1+t^{\lambda}}\left[\frac{c_{1} t^{\rho_{1}}}{\Gamma\left(1+\rho_{1}\right)}+\frac{c_{2} t^{\rho_{1}-\rho_{2}}}{\Gamma\left(1+\rho_{1}-\rho_{2}\right)}\right] \\
\leq & {\left[\frac{c_{1}}{\Gamma\left(1+\rho_{1}\right)}+\frac{c_{2}}{\Gamma\left(1+\rho_{1}-\rho_{2}\right)}\right]\|x-y\|_{E}, }
\end{aligned}
$$


which implies that the operator $F: E \rightarrow E$ is a contraction operator, so the Banach contraction principle assures that the operator $F$ has a unique fixed point $y_{T}(t) \in E$. According to some arguments above, we obtain that $x_{T}(t)=I_{0+}^{\rho_{2}} y_{T}(t)$ is one unique solution of the Equation (32) with zero initial value condition. Thus, according to Definition 5, we obtain that the problem (1) has one unique approximate solution.

Example 4. Now, we consider the initial value problem as following

$$
\left\{\begin{array}{l}
D_{0+}^{\frac{1}{2}+\frac{t}{200\left(1+t^{2}\right)}} x(t)=\frac{\Gamma\left(\frac{3}{2}\right) x^{4}}{12\left(1+t^{2}\right)^{4}\left(1+x^{4}\right)}+\frac{\Gamma\left(\frac{7}{6}\right)\left(D_{0+}^{\frac{1}{3}+\frac{t}{600\left(1+t^{2}+t^{3}\right)}} x\right)^{2}}{12\left(1+t^{2}\right)^{2}\left(1+\left(D_{0+}^{\left.\left.\frac{1}{3}+\frac{t}{600\left(1+t^{2}+t^{3}\right)} x\right)^{2}\right)}, 0<t<+\infty,\right.\right.} \\
x(0)=0 .
\end{array}\right.
$$

We let

$$
\begin{gathered}
p(t)=\frac{1}{2}+\frac{t}{200\left(1+t^{2}\right)}, q(t)=\frac{1}{3}+\frac{t}{600\left(1+t^{2}+t^{3}\right)}, 0 \leq t<+\infty \\
f(t, x(t), y(t))=\frac{\Gamma\left(\frac{3}{2}\right) x^{4}(t)}{12\left(1+t^{2}\right)^{4}\left(1+x^{4}(t)\right)}+\frac{\Gamma\left(\frac{7}{6}\right) y^{2}(t)}{12\left(1+t^{2}\right)^{2}\left(1+y^{2}(t)\right)}, 0<t<+\infty, x(t), y(t) \in R .
\end{gathered}
$$

Obviously, we get $\lim _{t \rightarrow+\infty} p(t)=\frac{1}{2}$ and $\lim _{t \rightarrow+\infty} q(t)=\frac{1}{3}$, thus, $p$ satisfies $\left(A_{1}\right)$ with $\rho_{1}=\frac{1}{2}$, $\rho_{2}=\frac{1}{3}$. That $f(t, 0,0)=0$. In addition, for all $0 \leq t<+\infty, x(t), y(t) \in R$, from the differentiation mean theorem, we get

$$
\begin{array}{ll} 
& \left|f\left(t,\left(1+t^{2}\right) x_{1},\left(1+t^{2}\right) y_{1}\right)-f\left(t,\left(1+t^{2}\right) x_{2},\left(1+t^{2}\right) y_{2}\right)\right| \\
\leq \quad & \frac{\Gamma\left(\frac{3}{2}\right)}{12}\left|\frac{x_{1}^{4}}{1+\left(1+t^{2}\right)^{4} x_{1}^{4}}-\frac{x_{2}^{4}}{1+\left(1+t^{2}\right)^{4} x_{2}^{4}}\right| \\
& \left.+\frac{\Gamma\left(\frac{7}{6}\right)}{12} \mid \frac{y_{1}^{2}(t)}{1+\left(1+t^{2}\right)^{2} y_{1}^{2}}-\frac{y_{2}^{2}}{1+\left(1+t^{2}\right)^{2} y_{2}^{2}}\right) \mid \\
\leq \quad & \frac{\Gamma\left(\frac{3}{2}\right)}{3}\left|x_{1}-x_{2}\right|+\frac{\Gamma\left(\frac{7}{6}\right)}{3}\left|y_{1}-y_{1}\right|,
\end{array}
$$

which implies that $f$ satisfies $\left(A_{2}\right)$ with $c_{1}=\frac{\Gamma\left(\frac{3}{2}\right)}{3}, c_{2}=\frac{\Gamma\left(\frac{7}{6}\right)}{3}$, which satisfies

$$
\begin{aligned}
& \frac{c_{1}}{\Gamma\left(1+\rho_{1}\right)}+\frac{c_{2}}{\Gamma\left(1+\rho_{1}-\rho_{2}\right)} \\
= & \frac{\Gamma\left(\frac{3}{2}\right)}{3} \frac{1}{\Gamma\left(1+\frac{1}{2}\right)}+\frac{\Gamma\left(\frac{7}{6}\right)}{3} \frac{1}{\Gamma\left(1+\frac{1}{2}-\frac{1}{3}\right)} \\
= & \frac{2}{3}<1 .
\end{aligned}
$$

For given arbitrary small $\varepsilon=\frac{1.1}{100}$, there exists $T=\frac{22}{\varepsilon}=2000$, such that

$$
\begin{gathered}
\left|p(t)-\frac{1}{2}\right|=\frac{t}{200\left(1+t^{2}\right)}<\frac{1}{t} \leq \frac{1}{T}=\frac{\varepsilon}{22}<\varepsilon, t \geq T, \\
\left|q(t)-\frac{1}{3}\right|=\frac{t}{600\left(1+t^{2}+t^{3}\right)}<\frac{1}{t} \leq \frac{1}{T}=\frac{\varepsilon}{22}<\varepsilon, t \geq T .
\end{gathered}
$$


Now, we consider function $p(t)$ restricted on interval $[0, T]=[0,2000]$. By the right continuity of function $p(t)$ at point 0 , for $\varepsilon=\frac{1.1}{100}$, taking $\delta_{0}=2$, when $0 \leq t \leq \delta_{0}=2$, we have

$$
\begin{gathered}
|p(t)-p(0)|=\left|\frac{t}{200\left(1+t^{2}\right)}\right| \leq \frac{t}{200}<\frac{\delta_{0}}{200}=\frac{1}{100}<\frac{1.1}{100}=\varepsilon . \\
|q(t)-q(0)|=\left|\frac{t}{600\left(1+t^{2}+t^{3}\right)}\right| \leq \frac{t}{600}<\frac{\delta_{0}}{200}=\frac{1}{100}<\frac{1.1}{100}=\varepsilon .
\end{gathered}
$$

We get $t_{1}=\delta_{0}=2$. By the right continuity of functions $p(t), q(t)$ at the point $t_{1}$, for $\varepsilon=\frac{1.1}{100}$, taking $\delta_{1}=2$, when $0 \leq t-t_{1} \leq \delta_{1}$, by differential mean value theorem, we have

$$
\begin{aligned}
\left|p(t)-p\left(t_{1}\right)\right| & =\left|\frac{t}{200\left(1+t^{2}\right)}-\frac{t_{1}}{200\left(1+t_{1}^{2}\right)}\right| \\
& \leq \quad\left|\frac{1-\xi^{2}}{200\left(1+\xi^{2}\right)^{2}}\right|\left|t-t_{1}\right| \\
& \leq \frac{1+\xi^{2}}{200\left(1+\xi^{2}\right)^{2}}\left|t-t_{1}\right| \\
& \leq \frac{1}{200}\left|t-t_{1}\right| \\
& <\frac{\delta_{1}}{200}=\frac{1}{100}<\frac{1.1}{100}=\varepsilon \\
\left|q(t)-q\left(t_{1}\right)\right| & =\left|\frac{t}{600\left(1+t^{2}+t^{3}\right)}-\frac{t_{1}}{600\left(1+t_{1}^{2}+t_{1}^{3}\right)}\right| \\
& \leq\left|\frac{1-\eta^{2}-2 \eta^{3}}{600\left(1+\eta^{2}+\eta^{3}\right)^{2}}\right| t-t_{1} \mid \\
& \leq\left|\frac{1+\eta^{2}+2 \eta^{3}}{600\left(1+\eta^{2}+\eta^{3}\right)^{2}}\right|\left|t-t_{1}\right| \\
& \leq \frac{3}{600}\left|t-t_{1}\right| \\
& <\frac{\delta_{1}}{200}=\frac{1}{100}<\frac{1.1}{100}=\varepsilon
\end{aligned}
$$

where $t_{1}<\xi<t, t_{1}<\eta<t$. We let $t_{2}=t_{1}+\delta_{1}=4$. By the right continuity of function $p(t)$ at point $t_{2}$, for $\varepsilon=\frac{1.1}{100}$, taking $\delta_{2}=2$, when $0 \leq t-t_{1} \leq \delta_{2}$, by the same reasons above, we have

$$
\begin{gathered}
\left|p(t)-p\left(t_{1}\right)\right|=\left|\frac{t}{200\left(1+t^{2}\right)}-\frac{t_{2}}{20\left(1+t_{2}^{2}\right)}\right|<\frac{\delta_{2}}{200}=\frac{1}{100}<\frac{1.1}{100}=\varepsilon, \\
\left|q(t)-q\left(t_{1}\right)\right|=\left|\frac{t}{600\left(1+t^{2}+t^{3}\right)}-\frac{t_{2}}{600\left(1+t_{2}^{2}+t^{3}\right)}\right|<\frac{\delta_{2}}{200}=\frac{1}{100}<\frac{1.1}{100}=\varepsilon,
\end{gathered}
$$

Continuing this procession, from $t_{n-1}=2(n-1)<2000, t_{n}=t_{n-1}+\delta_{n-1}=2(n-1)+2=2000$, we get $n=1000$. Thus, let

$$
p_{1} \doteq p(0)=\frac{1}{2}, p_{2} \doteq p\left(t_{1}\right)=p(2)=\frac{1}{2}+\frac{2}{200(1+4)},
$$




$$
\begin{gathered}
p_{3} \doteq p\left(t_{2}\right)=p(4)=\frac{1}{2}+\frac{4}{200 \times(1+16)}, \cdots, p_{1000}=p\left(t_{999}\right)=p(1998)=\frac{1}{2}+\frac{1998}{200 \times\left(1+1998^{2}\right)} . \\
q_{1} \doteq q(0)=\frac{1}{3}, q_{2} \doteq q\left(t_{1}\right)=q(2)=\frac{1}{3}+\frac{2}{600(1+4+8)}, \\
q_{3} \doteq q\left(t_{2}\right)=q(4)=\frac{1}{3}+\frac{4}{600 \times(1+16+64)}, \cdots, \\
q_{1000}=q\left(t_{999}\right)=q(1998)=\frac{1}{3}+\frac{1998}{600 \times\left(1+1998^{2}+1998^{3}\right)} .
\end{gathered}
$$

As a result, we get intervals $[0,2],(2,4], \ldots,(1998,2000],(2000,+\infty)$ and function $\alpha(t)$ defined by

$$
\begin{aligned}
& \alpha(t)=\left\{\begin{array}{l}
p_{1}=\frac{1}{2}, \text { for } t \in[0,2], \\
p_{2}=\frac{1}{2}+\frac{2}{200 \times(1+4)}, \text { for } t \in(2,4] \\
p_{3}=\frac{1}{2}+\frac{4}{200 \times(1+16)}, \text { for } t \in(4,6] \\
\cdots, \\
p_{1000}=\frac{1}{2}+\frac{1998}{200 \times\left(1+1998^{2}\right)}, \text { for } t \in(1998,2000] \\
\rho_{1}=\frac{1}{2}, \quad \text { for } t \in(2000,+\infty) .
\end{array}\right. \\
& \beta(t)=\left\{\begin{array}{l}
q_{1}=\frac{1}{3}, \text { for } t \in[0,2], \\
q_{2}=\frac{1}{3}+\frac{2}{600 \times(1+4+8)}, \text { for } t \in(2,4], \\
q_{3}=\frac{1}{3}+\frac{4}{600 \times(1+16+64)}, \text { for } t \in(4,6], \\
\cdots, \\
q_{1000}=\frac{1}{3}+\frac{1998}{6000 \times\left(1+1998^{2}+1998^{3}\right)}, \text { for } t \in(1998,2000] \\
\rho_{2}=\frac{1}{3}, \quad \text { for } t \in(2000,+\infty) .
\end{array}\right.
\end{aligned}
$$

By Definitions 4 and 5 and the arguments of Theorem 1, the problem (43) has one unique approximate solution.

Remark 1. From Lemma 6 and Definition 5, we may take arbitrary small $\varepsilon$, such that the problem (43) has one unique approximate solution. This means that the proximity is very high.

Example 5. Finally, we calculate the approximate solution of the following initial value problem for linear equation

$$
D_{0+}^{\frac{1}{2}+\frac{t}{200\left(1+t^{2}\right)}} x(t)=t^{\frac{1}{4}}, x(0)=0,0<t<+\infty,
$$

According to analysis in Example 4, we get intervals [0,2], $(2,4], \ldots,(1998,2000],(2000,+\infty)$ and function $\alpha(t)$ defined in (44). By Definitions 4 and 5 , we calculate out the approximate solution of the problem (45) as following 


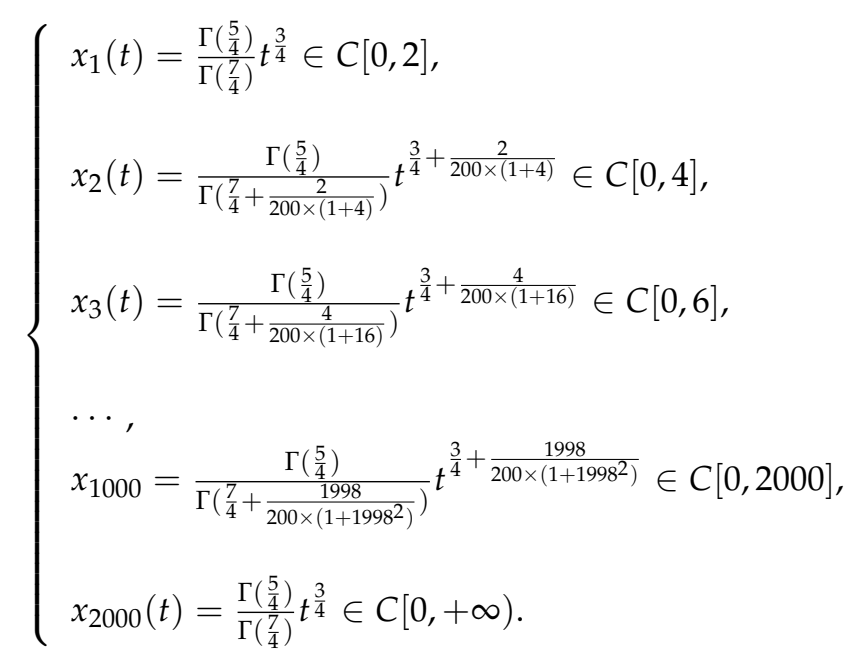

Remark 2. By the characters of variable order derivative, we cannot get accurate solution of the problem (45). Hence, the approximate solution given by us is significative.

\section{Conclusions}

In this paper, we have obtained the unique existence result of approximate solution of initial value problem for fractional differential equation of variable order involving with the variable order derivative defined on the half-axis. Through discussing the characters of variable order calculus(integral and derivative), we introduce the concept of approximate solution to the problem. Based on our discussion and analysis, using the fixed point theorem, we have found the unique existence results. As applications, two examples are presented to illustrate the main results. The issue of the existence and qualitative analysis of approximate solution of initial value problems for fractional differential equation of variable order is interesting. In the future, we will consider the existence and qualitative analysis of approximate solution of initial value problem for singular fractional differential equation of variable order.

Author Contributions: All authors contributed equally in writing this article. All authors read and approved the final manuscript.

Funding: This research is funded by the National Natural Science Foundation of China (11671181). This research is also supported by Doctor Science Foundation of Shandong Jiaotong University and Shandong Jiaotong University "Climbing" Research Innovation Team Program.

Conflicts of Interest: The authors declare no conflict of interest.

\section{References}

1. Valério, D.; da Costa, J.S. Variable-order fractional derivative and their numerical approximations. Signal Process. 2011, 91, 470-483. [CrossRef]

2. Tavares, D.; Almeida, R.; Torres, D.F.M. Caputo derivatives of fractional variable order: Numerical approximations. Commun. Nonlinear Sci. Numer. Simul. 2016, 35, 69-87. [CrossRef]

3. Razminia, A.; Dizaji, A.F.; Majd, V.J. Solution existence for non-autonomous variable-order fractional differential equations. Math. Comput. Model. 2012, 55, 1106-1117. [CrossRef]

4. Alikhanov, A.A. Boundary value problems for the equation of the variable order in differential and difference settings. Appl. Math. Comput. 2012, 219, 3938-3946. [CrossRef]

5. Moghaddam, B.P.; Machado, J.A.T.; Behforooz, H. An integro quadratic spline approach for a class of variable-order fractional initial value problems. Chaos Solitons Fractals 2017, 102, 354-360. [CrossRef]

6. Zúniga-Aguilar, C.J.; Romero-Ugalde, H.M.; Gómez-Aguilar, J.F.; Escobar-Jiménez, R.F.; Valtierra-Rodríguez, M. Solving fractional differential equations of variable-order involving operator with Mittag-Leffler kernel using artifical neural networks. Chaos Solitons Fractals 2017, 103, 382-403. [CrossRef] 
7. Chen, C.M.; Liu, F.; Anh, V.; Turner, I. Numberical schemes with high spatial accuracy for a variable-order anomalous subdiffusion equation. SIAM J. Sci. Comput. 2010, 32, 1740-1760. [CrossRef]

8. Sierociuk, D.; Malesza, W.; Macias, M. Derivation, interpretation, and analog modelling of fractional variable order derivative definition. Appl. Math. Model. 2015, 39, 3876-3888. [CrossRef]

9. Sun, H.; Chen, W.; Wei, H.; Chen, Y. A comparative study of constant-order and variable-order fractional models in characterizing memory property of systems. Eur. Phys. J. Spec. Top. 2011, 193, 185-192. [CrossRef]

10. Ye, H.; Gao, J.; Ding, Y. A generalized Gronwall inequality and its application to a fractional differential equation. J. Math. Anal. Appl. 2007, 328, 1075-1081. [CrossRef]

11. Sousa, J.V.D.C.; de Oliveira, E.C. Two new fractional derivatives of variable order with non-singular kernel and fractional differential equation. Comput. Appl. Math. 2018, 37, 5375-5394. [CrossRef]

12. Gómez-Aguilar, J.F. Analytical and numerical solutions of nonlinear alcoholism model via variable-order fractional differential equations. Phys. A 2018, 494, 52-57. [CrossRef]

13. Yang, J.; Yao, H.; Wu, B. An efficient numberical method for variable order fractional functional differential equation. Appl. Math. Lett. 2018, 76, 221-226. [CrossRef]

14. Hajipour, M.; Jajarmi, A.; Baleanu, D.; Sun, H. On an accurate discretization of a variable-order fractional reaction-diffusion equation. Commun. Nonlinear Sci. Numer. Simul. 2019, 69, 119-133. [CrossRef]

15. Samko, S.G. Fractional integration and differentiation of variable order. Anal. Math. 1995, 21, $213-236$. [CrossRef]

16. Samko, S.G.; Boss, B. Integration and differentiation to a variable fractional order. Integral Transform. Spec. Funct. 1993, 1, 277-300. [CrossRef]

17. Zhang, S.; Li, S.S.; Hu, L. The existeness and uniqueness result of solutions to initial value problems of nonlinear diffusion equations involving with the conformable variable derivative. Rev. R. Acad. Cienc. Exactas Fís. Nat. Ser. A Math. RACSAM 2018. [CrossRef]

18. Zhang, $\mathrm{S}$. The uniqueness result of solutions to initial value problem of differential equations of variable-order. Rev. R. Acad. Cienc. Exactas Fís. Nat. Ser. A Math. RACSAM 2018, 112, 407-423. [CrossRef]

19. Malesza, W.; Macias, M.; Sierociuk, D. Analysitical solution of fractional variable order differential equations. J. Comput. Appl. Math. 2019, 348, 214-236. [CrossRef]

20. Kian, Y.; Sorsi, E.; Yamamoto, M. On time-fractional diffusion equations with space-dependent variable order. Ann. Henri Poincaré 2018, 19, 3855-3881. [CrossRef]

21. Kilbas, A.A.; Srivastava, H.M.; Trujillo, J.J. Theory and Applications of Fractional Differential Equations; Elsevier: Amsterdam, The Netherlands, 2006.

22. Kou, C.; Zhou, H.; Yan, Y. Existence of solutions of initial value problems for nonlinear fractional differential equations on the half-axis. Nonlinear Anal. 2011, 74, 5975-5986. [CrossRef]

23. Deng, J.; Deng, Z. Existence of solutions of initial value problems for nonlinear fractional differential equations. Appl. Math. Lett. 2014, 32, 6-12. [CrossRef]

24. Agarwal, R.P.; Benchohra, M.; Hamani, S. Boundary value problems for fractional differential equations. Georgian Math. J. 2009, 16, 401-411.

25. Dong, X.; Bai, Z.; Zhang, S. Positive solutions to boundary value problems of p-Laplacian with fractional derivative. Bound. Value Probl. 2017, 5, 1-15. [CrossRef]

26. Bai, Z.; Zhang, S.; Sun, S.; Chun, Y. Monotone iterative method for a class of fractional differential equations. Electron. J. Differ. Equ. 2016, 6, 1-8.

(C) 2019 by the authors. Licensee MDPI, Basel, Switzerland. This article is an open access article distributed under the terms and conditions of the Creative Commons Attribution (CC BY) license (http://creativecommons.org/licenses/by/4.0/). 